\title{
The BBX gene family in Moso bamboo (Phyllostachys edulis): identification, characterization and expression profiles
}

\author{
Ruifang Ma ${ }^{1,2}$, Jialu Chen ${ }^{1,2}$, Bin Huang ${ }^{1,2}$, Zhinuo Huang ${ }^{1,2}$ and Zhijun Zhang ${ }^{1,2^{*}}$
}

\begin{abstract}
Background: The BBX (B-box) family are zinc finger protein (ZFP) transcription factors that play an essential role in plant growth, development and response to abiotic stresses. Although BBX genes have been characterized in many model organisms, genome-wide identification of the BBX family genes have not yet been reported in Moso bamboo (Phyllostachys edulis), and the biological functions of this family remain unknown.

Result: In the present study, we identified 27 BBX genes in the genome of Moso bamboo, and analysis of their conserved motifs and multiple sequence alignments revealed that they all shared highly similar structures.

Additionally, phylogenetic and homology analyses indicated that PeBBX genes were divided into three clusters, with whole-genome duplication (WGD) events having facilitated the expansion of this gene family. Light-responsive and stress-related cis-elements were identified by analyzing cis-elements in the promoters of all PeBBX genes. Short time-series expression miner (STEM) analysis revealed that the PeBBX genes had spatiotemporal-specific expression patterns and were likely involved in the growth and development of bamboo shoots. We further explored the downstream target genes of PeBBXs, and GO/KEGG enrichment analysis predicted multiple functions of BBX target genes, including those encoding enzymes involved in plant photosynthesis, pyruvate metabolism and glycolysis/ gluconeogenesis.
\end{abstract}

Conclusions: In conclusion, we analyzed the PeBBX genes at multiple different levels, which will contribute to further studies of the BBX family and provide valuable information for the functional validation of this family.

Keywords: Moso bamboo, Gene family, Expression analysis, Characterization

\section{Background}

Transcription factors (TFs) are essential regulatory proteins that can activate or repress the expression of their target genes and thus play an important regulatory role in all eukaryotes [1]. This regulation is achieved through specific interactions between the DNA-binding domain of the TF and the promoter of the target gene. These interactions are affected by TF transcriptional activation

\footnotetext{
* Correspondence: zjzhang@zafu.edu.cn

${ }^{1}$ State Key Laboratory of Subtropical Forest Cultivation, Zhejiang A\&F University, Lin'an, Zhejiang 311300 Hangzhou, China

${ }^{2}$ School of Forestry and Biotechnology, Zhejiang A\&F University, Lin'an,

Zhejiang 311300 Hangzhou, China
}

domains, oligomerization sites, nuclear localization signals and DNA binding domains [2-4]. Zinc finger TF members are divided into several subfamilies, based on their structural and functional characteristics. Among them, B-box (BBX) zinc finger proteins have received considerable attention in recent years due to their diverse functions [5].

BBX are a class of zinc finger protein TFs with one or two BBX structural domains, with some members also possessing CCT (conserved carboxy-terminal) structural domains. The most distinctive feature of BBX TFs is the presence of one BBX at the $\mathrm{N}$-terminus or two $\mathrm{BBX}$ modules in tandem, sometimes with a CCT

C The Author(s). 2021 Open Access This article is licensed under a Creative Commons Attribution 4.0 International License, which permits use, sharing, adaptation, distribution and reproduction in any medium or format, as long as you give appropriate credit to the original author(s) and the source, provide a link to the Creative Commons licence, and indicate if changes were made. The images or other third party material in this article are included in the article's Creative Commons licence, unless indicated otherwise in a credit line to the material. If material is not included in the article's Creative Commons licence and your intended use is not permitted by statutory regulation or exceeds the permitted use, you will need to obtain permission directly from the copyright holder. To view a copy of this licence, visit http://creativecommons.org/licenses/by/4.0/ The Creative Commons Public Domain Dedication waiver (http://creativecommons.org/publicdomain/zero/1.0/) applies to the data made available in this article, unless otherwise stated in a credit line to the data. 
(CONSTANS, CCMike and TOC) domain, at the Cterminus [6]. The BBX structure consists of 1-2 BBX modules with a length of 40 amino acid residues. Based on the similar sequences of the two modules, and the spacing characteristics with zinc-binding residues, BBXs can be classified into two types: BBX 1 (B1) and BBX 2 (B2). The zinc finger type of $\mathrm{B} 1$ is $\mathrm{C}-\mathrm{X} 2-\mathrm{C}-\mathrm{X} 7-8-\mathrm{C}-\mathrm{X} 2-$ D-X-A-X-L-C-X2-C- D-X3-HB, while the zinc finger type of B2 is C-X2-C-X3-P-X4-C-X2-D-X3-L-C-X2-CD-X3-H [7, 8].

The BBX proteins have unique tertiary structures that are stabilized by binding $\mathrm{Zn}$ ions [9]. Studies have shown that the BBX domain regulates protein-protein interactions as well as transcriptional processes [10-12]. BBX proteins have been shown to affect seedling development by integrating plant phytochromes and light signals sensed by cryptic photoreceptors through the COP1 and HY5 signaling pathways. In addition, some BBX proteins are involved in the photoperiodic pathway of flowering $[13,14]$. Sequence alignment analysis of BBX proteins suggests that the CCT structural domain of some BBX proteins are also highly conserved and functional [6], typically consisting of 42-43 amino acids [15]. Both Arabidopsis and rice each possess 17 members that have a CCT domain at their C-terminus, which plays a vital role in transcriptional regulation and nuclear protein transport $[16,17]$. The Arabidopsis regulator of photoperiodic flowering CO (CONSTAVS), or BBX1, regulates FT (FLOWERING LOCUS T) expression to control the flowering pathway by acting directly on the promoter of FT [15]. In addition, the CCT structural domain includes nuclear localization signals, which play an active role in the cytosolic localization of BBX TFs $[18,16,19]$.

Plants adapt to various environmental changes by altering their gene regulatory networks [20]. BBX TFs are induced in response to different abiotic stresses and are important candidates for enhancing abiotic stress tolerance in plants. In Arabidopsis, COLI (BBX2) is consistently up-regulated during long periods of cold acclimation [21] and BBX32 in Arabidopsis is responsive to methyl jasmonate (MeJA) hormone stress. In chrysanthemum, CmBBX24 improves tolerance to abiotic stresses, especially drought stress and low-temperature stress [22]. In banana, transcripts of the MaCOL1 gene were shown to accumulate rapidly under cold treatment [23].

Bamboo is one of the most important non-timber forest products globally [24]. Moso bamboo (Phyllostachys edulis, $P$. edulis) is fast-growing, easy to establish, and has both ecological and social benefits [25]. Due to these beneficial features, bamboo is widely used for wood, paper, artwork and food [26, 27]. In China, several species of bamboo are grown for dual use, with shoots being harvested for consumption while mature wood is used for timber [28].
The development and distribution of Moso bamboo are limited by unfavorable climatic and environmental conditions [29]. Genomic studies have the potential to result in dramatic increases in plant stress tolerance [30], but the previously published genome of Moso bamboo has not been sufficiently explored for this purpose. The new assembly of the Moso bamboo genome offers an opportunity to improve our understanding of the abundance, distribution and expansion of bamboo $B B X$ genes [31, 32]. In this study, we identified 27 genes encoding of Moso bamboo BBX family $(P e B B X)$ and investigated their structural domains, amplification patterns, and evolutionary relationships.

\section{Results}

\section{Identification and characterization of PeBBX genes in $P$. edulis}

Putative $B B X$ candidate genes were obtained from an HMMER3 search of the bamboo protein database using the plant BBX-type model (Pfam PF00643) with an Evalue threshold of $\leq 10^{-5}$. We removed redundant genes and verified the presence of conserved domains and motifs to arrive at a final set of $27 B B X$ family members. The genes were renamed PeBBX01-PeBBX27 based on their positions on chromosomal scaffolds (Table 1). Proteins encoded by the $27 \mathrm{PeBBX}$ genes contained 70 (PeBBX09) to 446 (PeBBX17) amino acids, and their MWs ranged from 7165.31 (PeBBX09) to 48231.99 (PeBBX17). Their predicted pIs ranged from 4.91 (PeBBX04/PeBBX13) to 8.49 (PeBBX15). Aliphatic amino acid indices showed that the thermal stability of the proteins ranged from 55.59 to 84.00 , indicating that differences in their thermal stability were relatively minor. The grand average of hydropathicity (GRAVY) scores of all BBX proteins were negative, suggesting that they were hydrophilic proteins. We predicted the subcellular localization of the proteins encoded by the $P e B B X$ genes and found that all were likely to localize to the nucleus.

\section{Phylogenetic analysis}

Previous studies have shown that $O s B B X$ genes can be divided into five subfamilies [9]. A NJ phylogenetic tree was constructed based on BBX protein sequences from bamboo, rice and $A$. thaliana to demonstrate the phylogenetic relationships between Moso bamboo and Gramineae plants as well as dicotyledonous plants (Additional file 4: Table S1). The sequences of $A$. thaliana were found to be distributed in subclade I, subclade II and subclade IV. However, in subclade III and subclade V, only sequences of $A$. thaliana and rice were found. In subclade IV, Moso bamboo had the highest number of PeBBX sequences, while subclade I and subclade II contained five PeBBX sequences. Moreover, the BBX of 


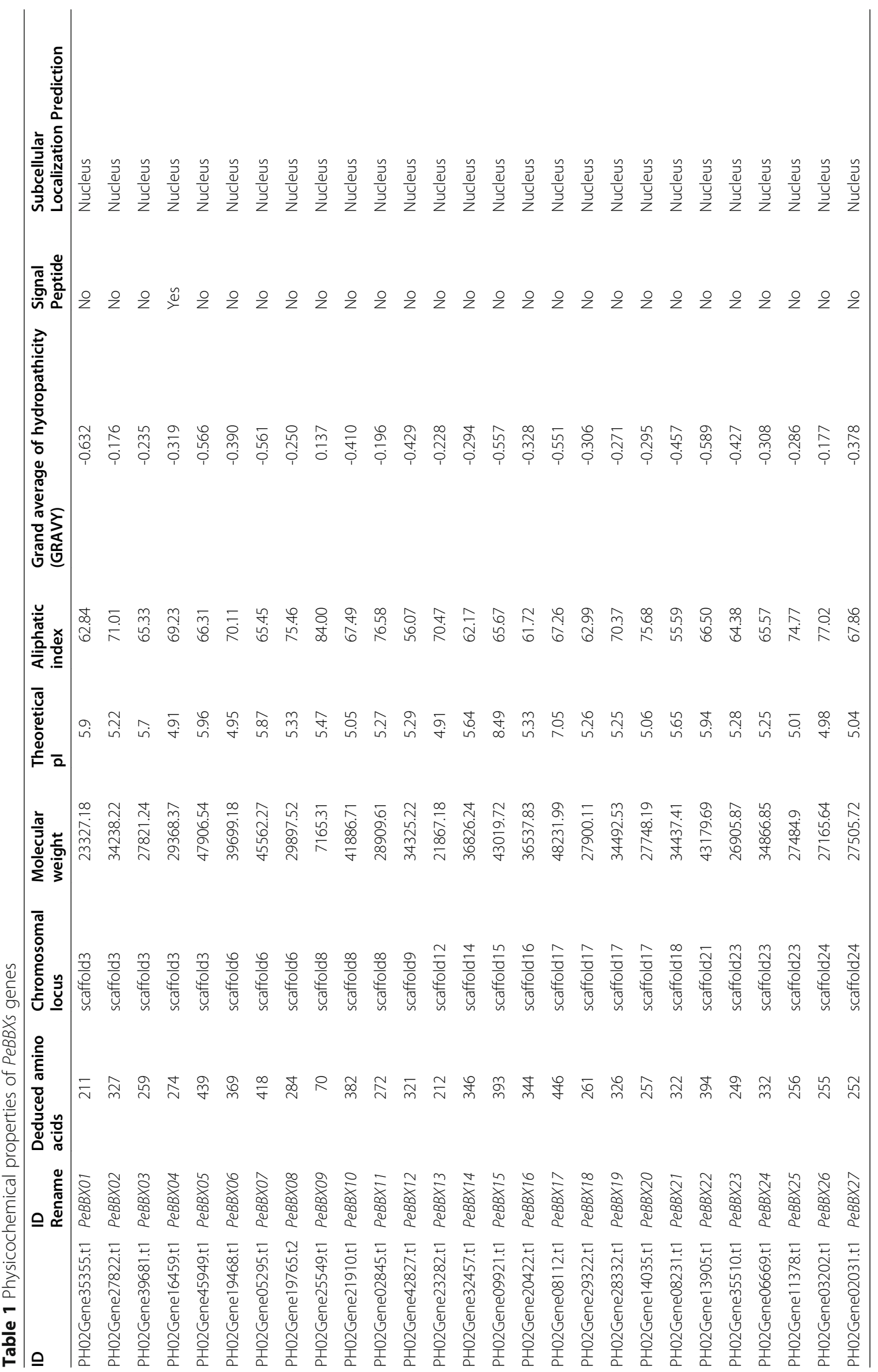


Moso bamboo and the BBX of rice clustered in one group (Fig. 1) and were more closely related.

\section{Gene structure, conserved domains, motifs and sequence analysis}

To gain insight into the structural features of $P e B B X$ genes in Moso bamboo, we compared their genomic DNA sequences to determine the number of introns and exons within each gene. The gene structures of all 27 $P e B B X$ genes were shown in Fig. 2a. Subclade I and subclade II were found to have similar gene structures, with a short overall length and only one intron. After comparing the motifs of subclades I, II and IV, we found that motif 1 of subfamily IV and subfamily II was in the more $\mathrm{N}$-terminal position, while motif 2 of subfamily I was in the more N-terminal position (Fig. 2b). After combining these results with the analysis of conserved structural domains (Fig. 2c), it is clear that motif 3 formed the CCT structural domain, and motif 1 formed the BOX1 structural domain. In contrast, motif 2 formed BOX2 structural domain, and the BOX structural domain was composed of motif 1 and motif 2 together.

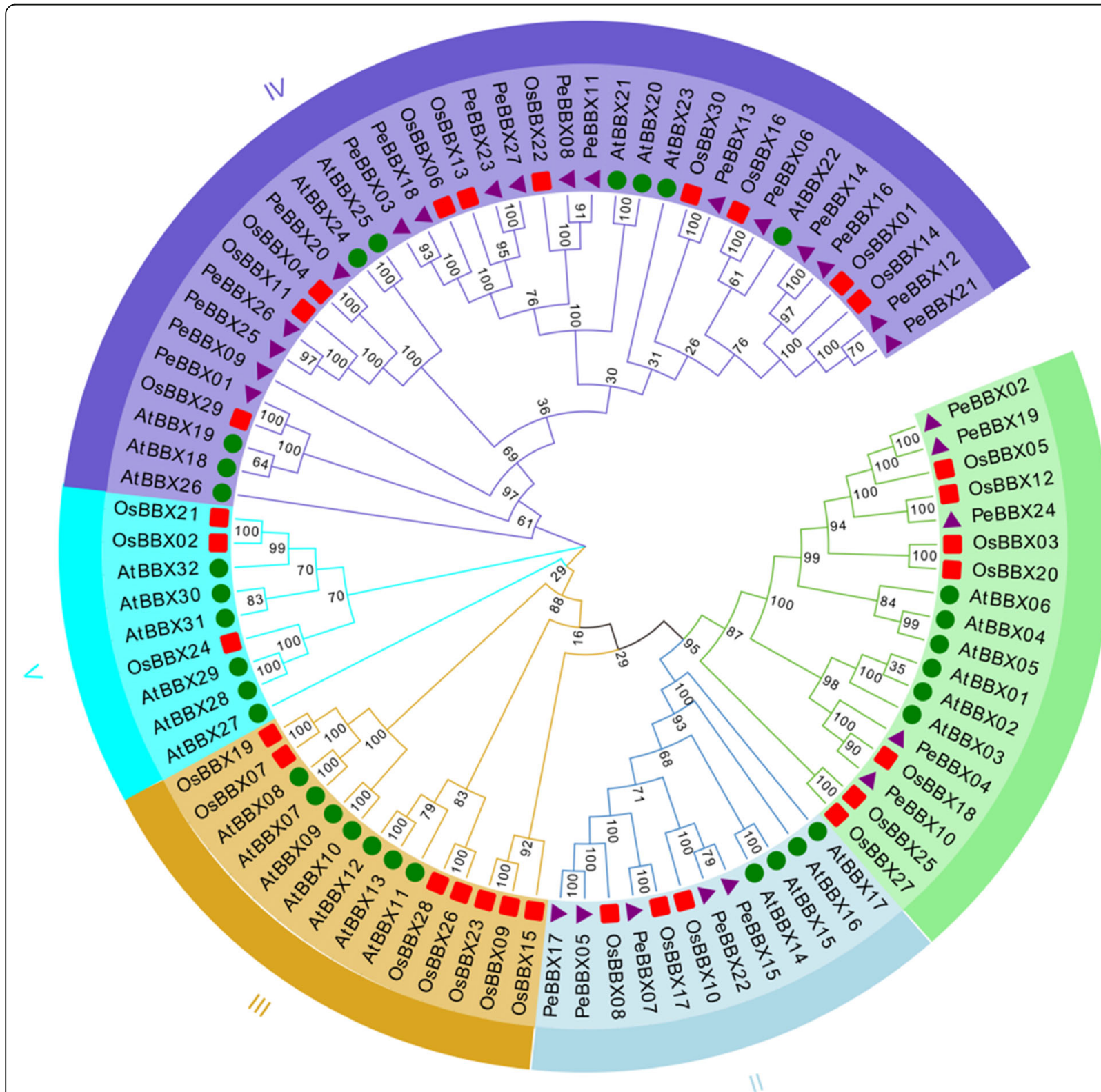

Fig. 1 Phylogenetic tree analysis of BBX sequences. The full-length amino acid sequences of 89 BBX proteins were used to construct the phylogenetic tree using MEGA7.0 with the neighbor-joining (NJ) method. The number at the branch represents the confidence value obtained by 100 bootstrap tests. AtBBX represents BBX protein sequence of Arabidopsis thaliana and OsBBX represents BBX protein sequence of rice 


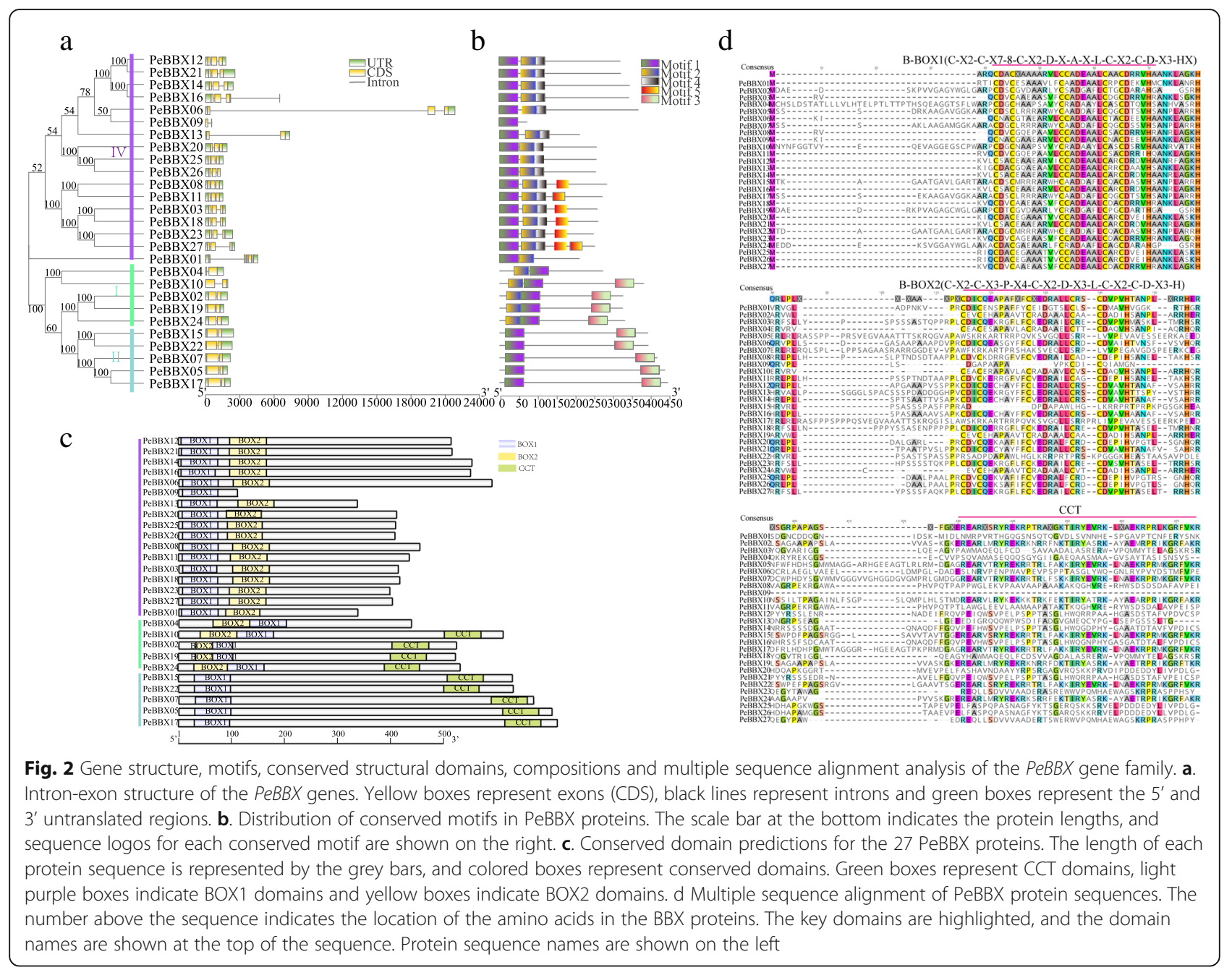

To analyze the sequence characteristics of PeBBX proteins, we used the CDD website to predict the structural domains in the full-length sequence of PeBBX proteins, revealing that the subclade IV had 17 members, nearly all of which had two BOX structural domains (BOX1 and BOX2). PeBBX09 was the only protein sequence in subclade IV that had only one BOX1 structural domain. In addition, the PeBBX sequences of the subclade II all possessed one BOX structural domain and one CCT structural domain. In contrast, the PeBBX sequences of the subclade IV all contained two BOX structural domains and one CCT structural domain, with the exception of PeBBX04.

To investigate the prevalence and locations of conserved protein domains, we created a multiple sequence alignment of $27 \mathrm{PeBBX}$ proteins. At the $\mathrm{N}$-terminus, all BBX family members contained a highly conserved BOX domain that consisted of approximately 30 amino acids (Fig. 2d). Some of the BBX sequences also had a CCT domain at the $\mathrm{C}$-terminus.

\section{Chromosomal location and gene duplication of PeBBX genes}

The $P e B B X$ genes were unequally distributed across the 11 chromosome scaffolds of Moso bamboo (Additional file 1: Figure S1). The largest number was found on scaffold 3 (5), followed by scaffold 17 (4), scaffolds 6 (3), 8 (3), 23 (3) and $2 P e B B X$ genes were mapped on scaffold 24. All other chromosomes contained a single $P e B B X$ gene. In all species, gene duplication events are prevalent, and they can produce new functional genes and drive the evolution of species. Therefore, we used MCScanX genomic co-frequency analysis to explore duplications within the $B B X$ gene family (Fig. 3a), revealing 30 segmental duplications in in the whole genome of Moso bamboo.

Monocotyledonous plants, including rice and maize, and herbaceous plants, including pepper and Arabidopsis, were selected for interspecific collinearity analysis by BLAST comparison of BBX homologous sequences. This analysis revealed that the $B B X$ genes in Moso bamboo 


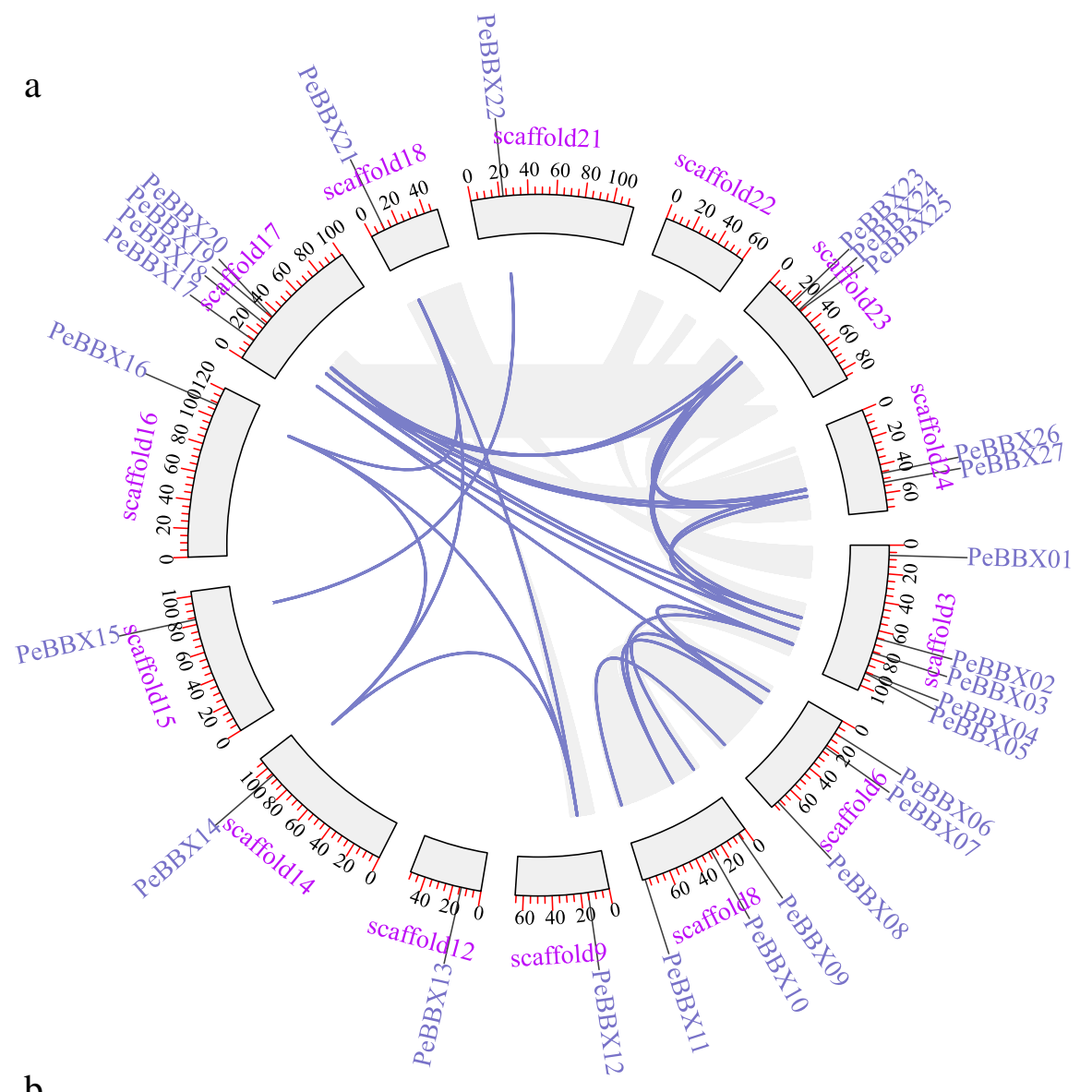

b

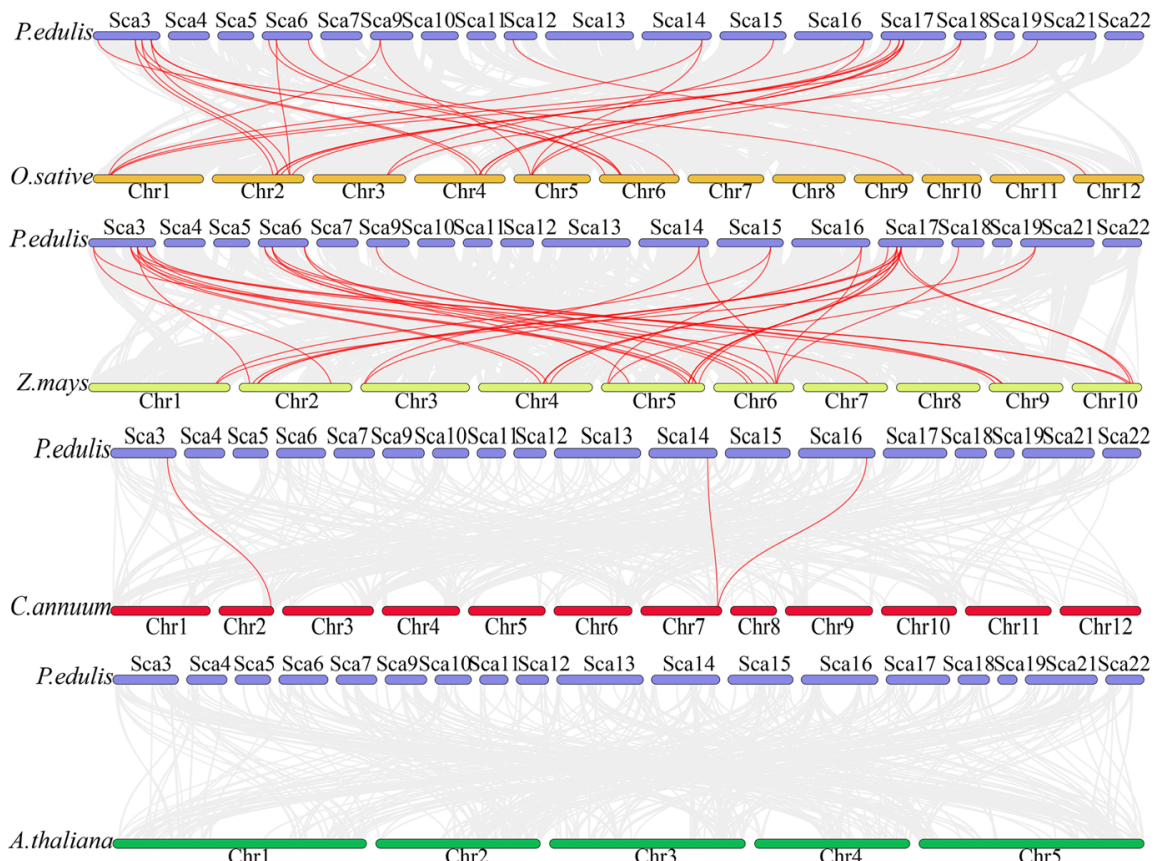

Fig. 3 (See legend on next page.) 
(See figure on previous page.)

Fig. 3 Chromosomal location and duplicated genes among PeBBX genes. a Intraspecific colinearity analysis. A total of 27 PeBBXs were mapped onto the chromosomes on the basis of their physical location. Chromosome numbers (scaffold1-scaffold24) are distributed in the outer circle, the blue lines indicate duplicated PeBBX gene pairs. b Analysis of collinearity between different species. The gray lines indicate duplicated blocks, while the red lines indicate duplicated BBX gene pairs. Chromosome numbers are at the bottom of each chromosome

and rice were highly homologous, indicating that they most likely had similar functions. In the model plant $A$. thaliana, no homology was identified with the $B B X$ genes of Moso bamboo. In addition, many highly homologous genes of $B B X$ in Moso bamboo were identified in maize, and three homologous genes were identified in pepper (Fig. 3b).

Based on the comparative sequence similarity of BBX family, among which 24 Moso bamboo homologous gene pairs were found, $\mathrm{Ka} / \mathrm{Ks}$ ratios of the 24 Moso bamboo $B B X$ gene pairs were calculated, and the Ks values were used to calculate the time of duplication events of Moso bamboo $B B X$ genes. We performed $\mathrm{Ka} / \mathrm{Ks}$ (evolutionary selection pressure) analysis on $24 \mathrm{PeBBX}$ homologous gene pairs in Moso bamboo, and found that among these 24 pairs of

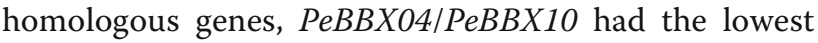
$\mathrm{Ka} / \mathrm{Ks}$ ratio (0.1734), followed by $\mathrm{PeBBX19/PeBBX02}$ (0.2422), while $P e B B X 20 / P e B B X 25$ had the highest ratio (0.4798). The $\mathrm{Ka} / \mathrm{Ks}$ ratio of all Moso bamboo $B B X$ homologs were less than 1 , indicating a strong purifying selection in the evolution of the Moso bamboo $B B X$ gene family (Table 2 ). The Ks values obtained during this analysis then used to calculate the duplication time of Moso bamboo $B B X$ genes during evolution. This analysis results showed that the replication time of Moso bamboo $B B X$ genes primarily occurred between 9 and 75 million years ago (mya), with nine gene pair replication events occurring in roughly 9-17 mya.

Table $2 \mathrm{Ka} / \mathrm{Ks}$ values of homologous PeBBX gene pairs

\begin{tabular}{|c|c|c|c|c|c|c|}
\hline Duplicate gene pair & $\mathrm{Ka}$ & Ks & $\mathrm{Ka} / \mathrm{Ks}$ & Purify Selection & Duplication type & Time $\left(\mathrm{Mya}^{\mathrm{a}}\right)$ \\
\hline PeBBX14/PeBBX16 & 0.042104596 & 0.152056047 & 0.276901819 & Yes & segmental & 11.69661899 \\
\hline PeBBX14/PeBBX21 & 0.19628168 & 0.448756769 & 0.437389905 & Yes & segmental & 34.51975143 \\
\hline PeBBX14/PeBBX12 & 0.182756627 & 0.446713424 & 0.4091138 & Yes & segmental & 34.36257111 \\
\hline PeBBX15/PeBBX22 & 0.052573616 & 0.202958669 & 0.259036072 & Yes & segmental & 15.6122053 \\
\hline PeBBX16/PeBBXX21 & 0.176648673 & 0.441908756 & 0.399740151 & Yes & segmental & 33.9929812 \\
\hline PeBBX16/PeBBX12 & 0.173011971 & 0.46856959 & 0.369234315 & Yes & segmental & 36.04381459 \\
\hline PeBBX17/PeBBX07 & 0.216882382 & 0.658188855 & 0.329513908 & Yes & segmental & 50.62991194 \\
\hline PeBBX18/PeBBX23 & 0.170545595 & 0.379913416 & 0.448906482 & Yes & segmental & 29.22410896 \\
\hline PeBBX19/PeBBX24 & 0.165046414 & 0.522000187 & 0.316180756 & Yes & segmental & 40.15386055 \\
\hline PeBBX20/PeBBX25 & 0.13529533 & 0.281953784 & 0.479849316 & Yes & segmental & 21.68875262 \\
\hline PeBBX17/PeBBX05 & 0.054743612 & 0.179281304 & 0.305350367 & Yes & segmental & 13.79086958 \\
\hline PeBBX18/PeBBX03 & 0.041517811 & 0.139739347 & 0.297108955 & Yes & segmental & 10.74918051 \\
\hline PeBBX19/PeBBX02 & 0.043371875 & 0.179063587 & 0.242214932 & Yes & segmental & 13.77412206 \\
\hline PeBBX18/PeBBX27 & 0.139056615 & 0.335375829 & 0.414629209 & Yes & segmental & 25.79814069 \\
\hline PeBBX20/PeBBXX26 & 0.136471916 & 0.295718856 & 0.461492101 & Yes & segmental & 22.74760428 \\
\hline PeBBX21/PeBBX 12 & 0.040608208 & 0.131673581 & 0.308400577 & Yes & segmental & 10.12873698 \\
\hline РeBBX27/PeBBX03 & 0.141112234 & 0.352596186 & 0.40020919 & Yes & segmental & 27.12278352 \\
\hline PeBBX24/PeBBX02 & 0.167787595 & 0.475482406 & 0.35287866 & Yes & segmental & 36.57556972 \\
\hline РeBBX23/PeBBXX3 & 0.165269986 & 0.429437165 & 0.384852546 & Yes & segmental & 33.03362806 \\
\hline PeBBX23/PeBBX27 & 0.051435606 & 0.126234197 & 0.40746174 & Yes & segmental & 9.710322865 \\
\hline РeBBX06/PeBBX09 & 0.067830454 & 0.194146225 & 0.349378174 & Yes & segmental & 14.93432503 \\
\hline PeBBX08/PeBBX11 & 0.056814836 & 0.148800797 & 0.381818089 & Yes & segmental & 11.44621513 \\
\hline PeBBX05/PeBBX07 & 0.205355044 & 0.547240162 & 0.375255799 & Yes & segmental & 42.09539711 \\
\hline PeBBX04/PeBBX10 & 0.16485542 & 0.950757381 & 0.173393784 & Yes & segmental & 73.13518318 \\
\hline
\end{tabular}




\section{Cis-element analysis of PeBBXs}

Sequence analysis of the promoter regions of $P e B B X$ genes via the PlantCARE website showed 431 associated cis-acting elements (Fig. 4). These included 152 cis-elements related to light response regulation. In addition, there were hormone-responsive elements, including Ethylene response element (ERE), abscisic acid (ABA) responsive elements (ABRE, ABA responsiveness) and MeJA acid responsive elements (CGTCA-motif and TGACG-motif). There were also other stress responsive elements, anaerobic response regulatory elements (ARE, anaerobic responsiveness), drought-related regulatory elements (MBS), low-temperature response elements (LTR, lowtemperature responsiveness), defense and stress responsiveness (TC-rich motif) and wounding and pathogen responsiveness (W-box). It was noteworthy that each gene had a unique composition of cis-acting elements (Additional file 2: Figure S2, Additional file 5: Table S2), with $P e B B X 06, P e B B X 13$ and $P e B B X 14$ containing more lightresponsive cis-elements, while $P e B B X 06$ and PeBBX14 contained more hormone-responsive elements.

\section{Transcript expression pattern analysis}

next quantified the expression patterns of the 27 PeBBX genes at different shoot heights using RNA-seq (Fig. 5, Additional file 6: Table S3). This analysis revealed that all 27 members had different expression patterns. PeBBX16 was highly expressed ubiquitously, while PeBBX21, PeBBX26, PeBBX08 and PeBBX27 were highly expressed at $0.2 \mathrm{~m}$. $P e B B X 19$ and $P e B B X 24$ showed a decreasing trend in expression at $4 \mathrm{~m}$. PeBBX17, PeBBX20 and PeBBX25 showed a positive correlation between their expression levels and the growth height of the shoots. The gene expression results showed that the PeBBX genes were expressed at all stages of shoot development.

STEM is a measurement of gene expression in similar samples at different time points to observe the changes in gene expression at each time point and elucidate the patterns of changes in interdependent relationships between genes. Temporal sequence analysis based on STEM was applied to the gene expression patterns of $P e B B X$ genes at multiple heights during the growth of

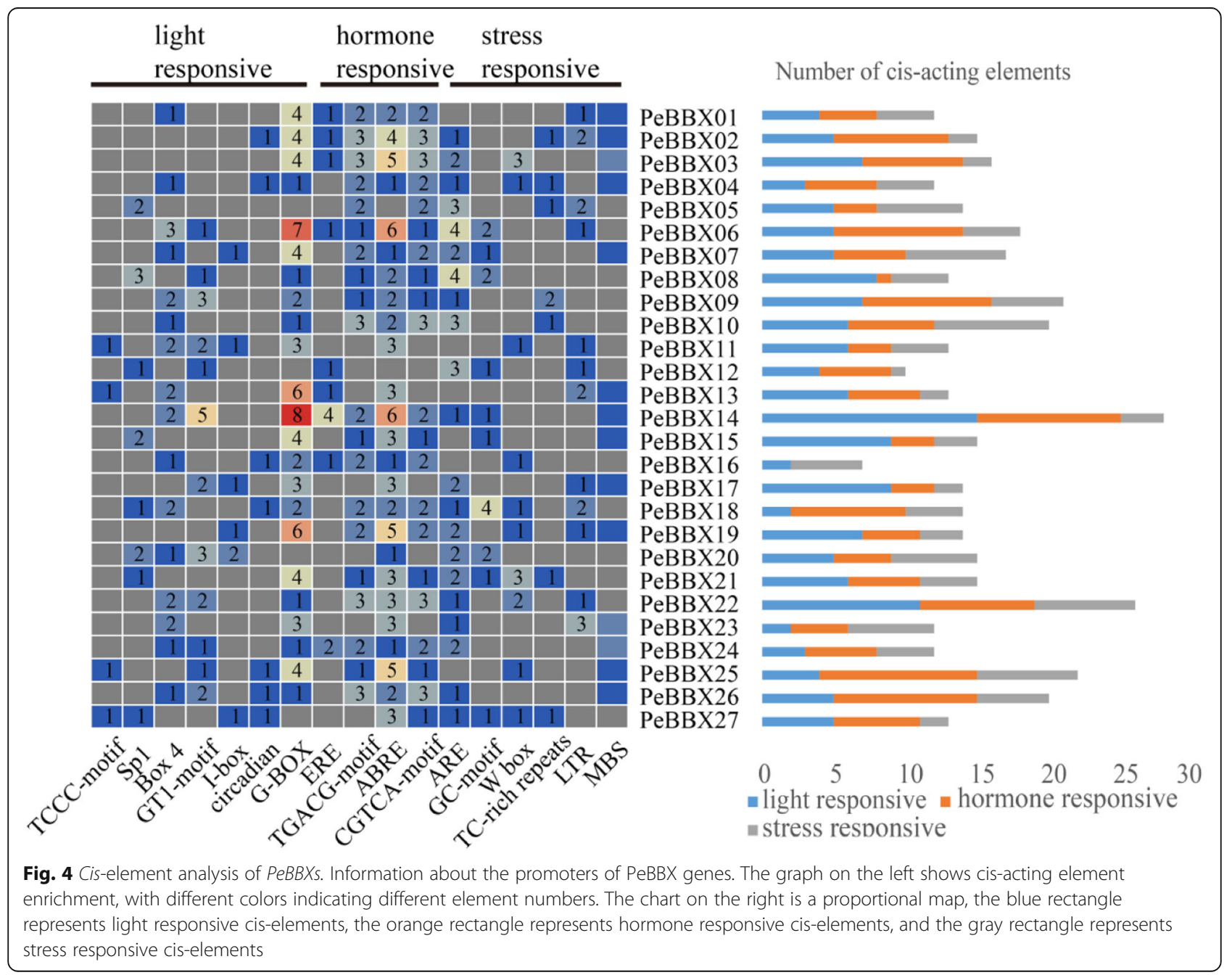




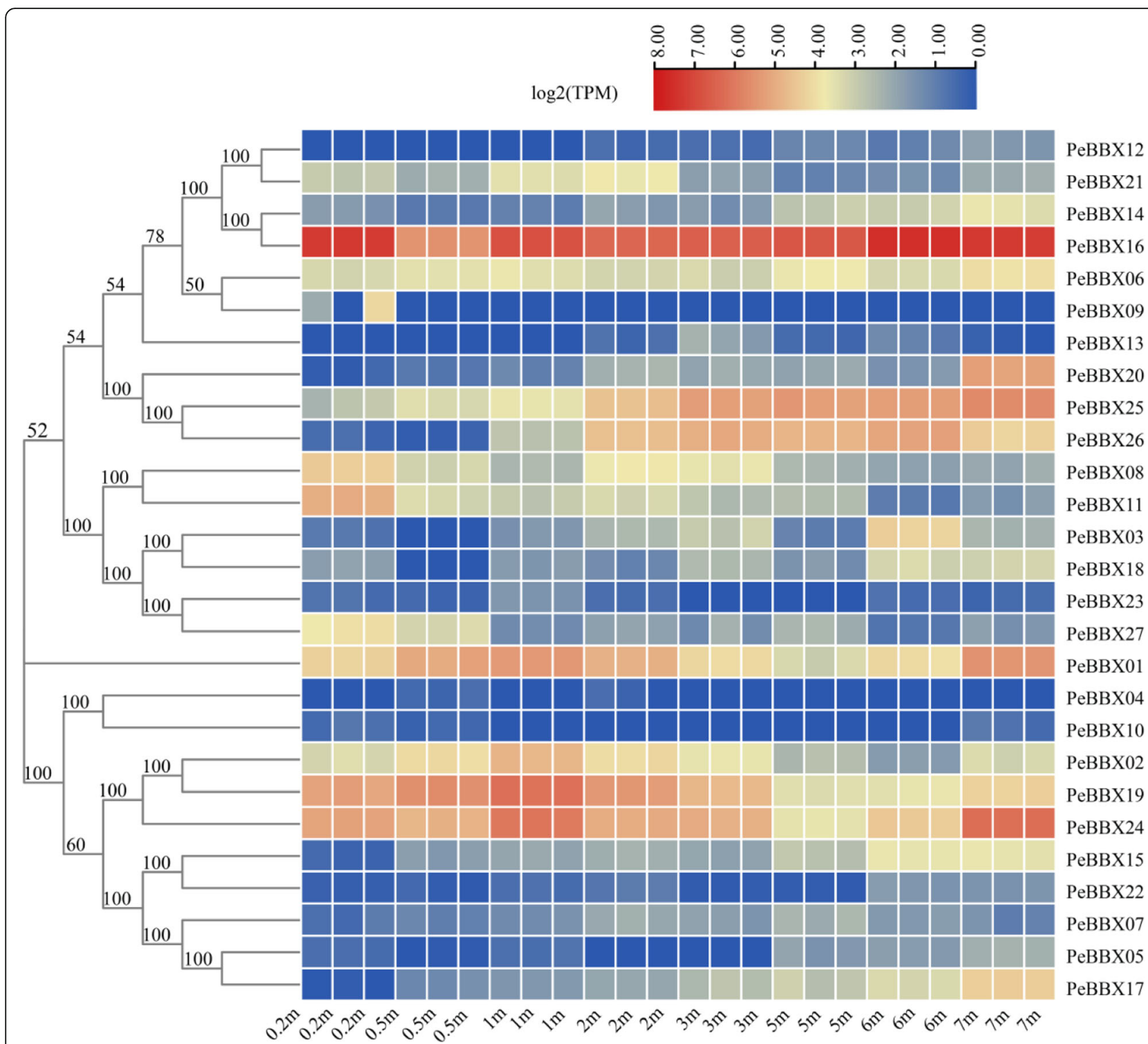

Fig. 5 Analysis of the expression patterns of different heights of bamboo shoots. That three replicates per condition. The relative expression levels are depicted according to the colour scale, where blue indicates low abundance and red indicates high abundance. Gene expression log 2 (TPM) was calculated as the sum of the abundance of all transcripts produced by a given gene

shoots, revealing 10 different gene expression profiles (Fig. 6a). Profile 9 contained $7 \mathrm{PeBBX}$ genes, which all had expression levels that were positively correlated with shoot height. Within this profile, PeBBX14, PeBBX15, $P e B B X 25$ and PeBBX26 had the highest expression at $5 \mathrm{~m}$, while $P e B B X 07$ had the highest expression at $4 \mathrm{~m}$ (Fig. 6b).

\section{Protein interaction network}

A protein interaction network was constructed using a network modelling method at STRING, its topological properties were analyzed at the network level. This analysis revealed that 14 proteins in the BBX family were predicted to interact with each other (Fig. 7). The PeBBX01 protein had the highest connectivity, interacting with 13 BBX proteins of Moso bamboo.

\section{PeBBX target gene identification, GO, KEGG annotation and analysis.}

We predicted BBX target genes using the JASPAR database and identified two BBX binding patterns (Fig. 8a), which were used to predict BBX target genes (Additional file 8). GO enrichment analysis and KEGG enrichment analysis of BBX target genes were also performed on GO enrichment tool (http://geneontology.org/page/goenrichment-analysis) and KEGG website, respectively. 


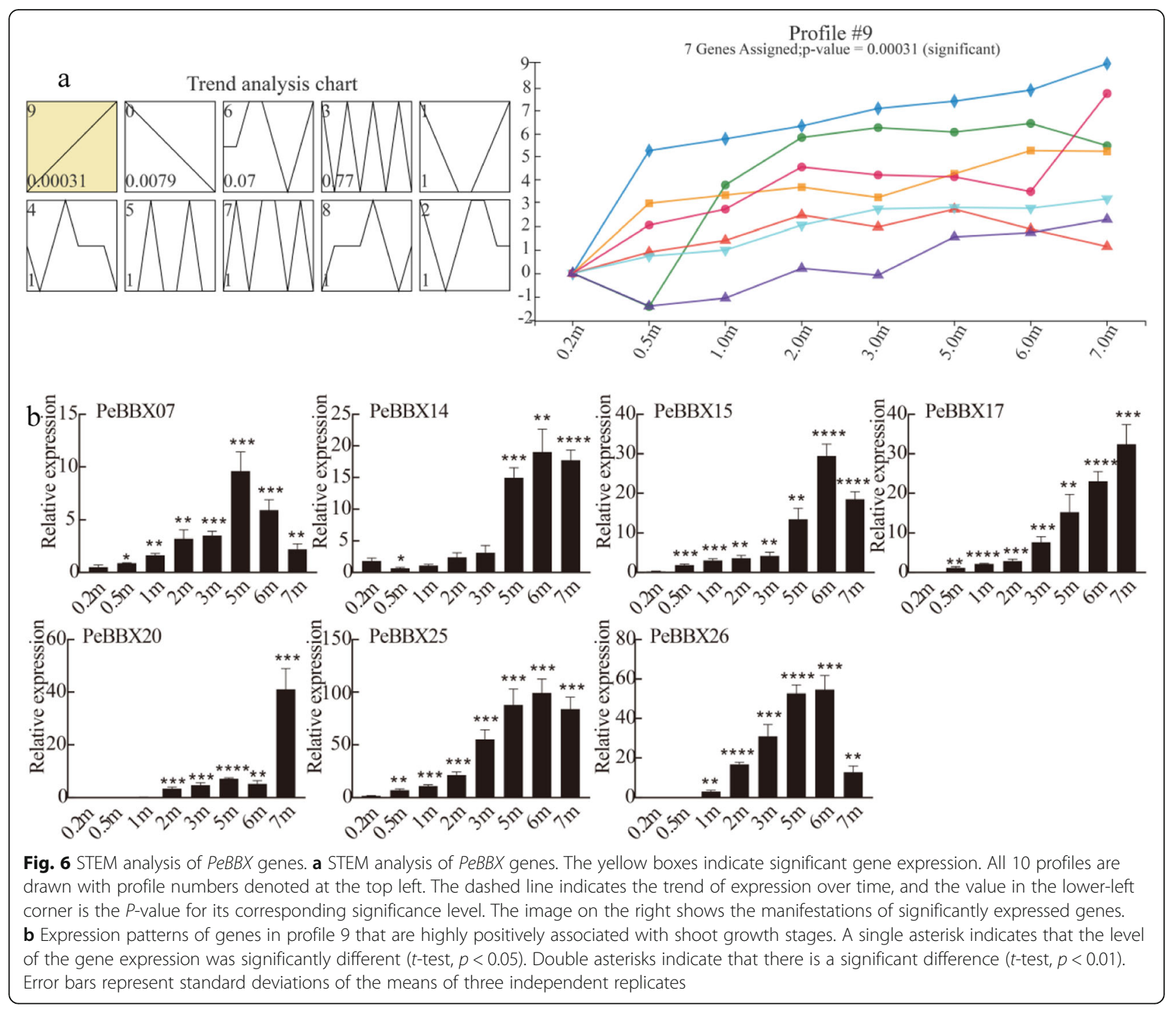

GO annotation enrichment was divided into three levels: biological process (BP), cellular component (CC) and molecular function (MF). A total of 185 target genes were predicted to be regulated by BBX members and were mainly enriched in categories falling under $\mathrm{CC}$ and $\mathrm{BP}$ (Fig. 8b). At the CC level, targets were primarily enriched in the Golgi apparatus (GO:0005794), and at the BP level, targets were mainly enriched in response to oxygen-containing compound (GO:1,901,700) and response to chemical (GO:0042221). At the MF level, there was only one enriched GO term: pigment binding (GO: 0031409). In addition, GO predicted that some BBX target genes were components of the photosystem complexes, including photosystem II (PSII) antenna complex, PSII associated light-harvesting complex II, light-harvesting complex and thylakoid light-harvesting complex (Additional file 9). The target genes included photopigment PR genes, such as PHO2Gene47533 and
PH02Gene42040, both of which are Chlorophyll a-b binding proteins (CP26). These targets indicated that $P e B B X$ genes were associated with the formation of complexes that is involved in the regulation of photosynthesis (Additional file 10). KEGG predicted that the major metabolic pathways enriched in BBX target genes were found to be primarily involved in Phenylpropanoid biosynthesis, Photosynthesis-antenna proteins, Pyruvate metabolism and Glycolysis/Gluconeogenesis (Fig. 8c, Additional file 11).

\section{Protein structure analysis by homology modeling}

To further analyze the protein function, the tertiary structures of the proteins were predicted. PeBBX04 from subclade I, PeBBX05 from subclade II and PeBBX13 from subclade IV were selected for tertiary structural homology modelling. The 3D structures of the three subclades were found to have different tertiary structures 


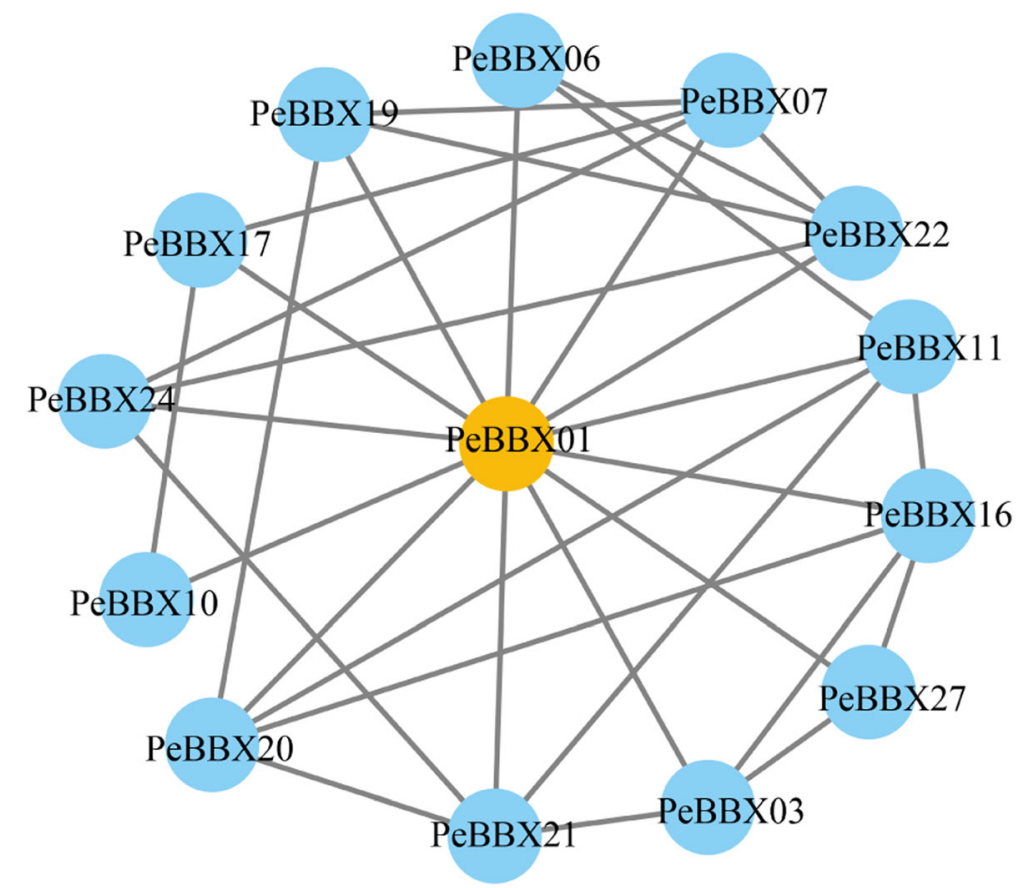

Fig. 7 Protein interaction network analysis. Nodes indicate proteins, and edges indicate the presence of interactions between two proteins. Nodes in orange indicate the highest level of connectivity for that protein

(Fig. 9). The PeBBX04 sequence consisted of two $\alpha$ helices and two $\beta$-folded lamellae and contained three amino acid residues that specifically bind to zinc ions (C.88, C.105, C.108). The PeBBX05 sequence consisted mainly of $\beta$-folded lamellae, and the PeBBX13 sequence was more complex, with four $\beta$-folded lamellae.

\section{Subcellular localization of BBX proteins}

PeBBX01 showed high connectivity in protein interaction network prediction, and to further understand the properties of this protein, we selected PeBBX01 for subcellular localization analysis. We constructed a transient expression MAS-PeBBX01-GFP vector for this gene for validation experimental purposes. The results showed that the MAS-GFP fluorescent signal in the control was dispersed throughout the tobacco cells, and the expressed fusion protein MAS-PeBBX01-GFP was specifically distributed in the nucleus (Fig. 10).

\section{Discussion}

Plants are often challenged with various unfavorable environmental conditions throughout their growth and development. In response to these environmental changes, a series of physiological and biochemical changes are produced. A diverse array of different genes are required for these changes, with TFs playing a central role [33, 34]. Zinc finger TFs are a superfamily of TFs with many members, including BBXs. In plants, several $B B X$ gene families have been identified, with 32 members in
Arabidopsis, 30 in rice, 29 in tomato, 30 in potato and 37 in pear $[2,9,6,35,36]$.

In this study, we identified a total of 27 genes encoding $\mathrm{BBX}$ proteins (PeBBX) in the Moso bamboo genome. The B-box1 and B-box2 structural domains of the 27 PeBBX protein sequences were highly conserved, and there was a strong similarity with the BBX sequences of rice and Arabidopsis. This indicated that the sequences of BBX were strongly conserved during the evolutionary process. The number of genes encoding specific TF families varies among plant species due to evolutionary expansion and the development of species-specific functions [37]. The results of the phylogenetic tree showed that PeBBXs lacks the III and V subclades, and expanded the subclade IV compared to Arabidopsis and rice. Among the BBX family members of Arabidopsis, 21 $\mathrm{BBX}$ have been functionally characterized. Among them, there were eight BBX proteins that were members of subclade IV and had either facilitative or repressive effects on photomorphogenesis. Recent analysis of BRrelated (brassinosteroid action-related) genes in bamboo has shown that the phytohormone BR regulated growth by promoting root cell division and elongation [38, 39], and $A T B B X 20$ in subcladeIV responded to the phytohormone BR [14]. In addition, these genes were involved in salt, cold, darkness, and hormone responses [14]. $O s B B X 14$, a member of rice subclade IV. Under photoperiodic conditions, OsBBX14 expression had a circadian rhythm and the $O s B B X 14$ overexpression strain 
$\mathrm{a}$ BBX binding pattern 1

BBX binding pattern 2

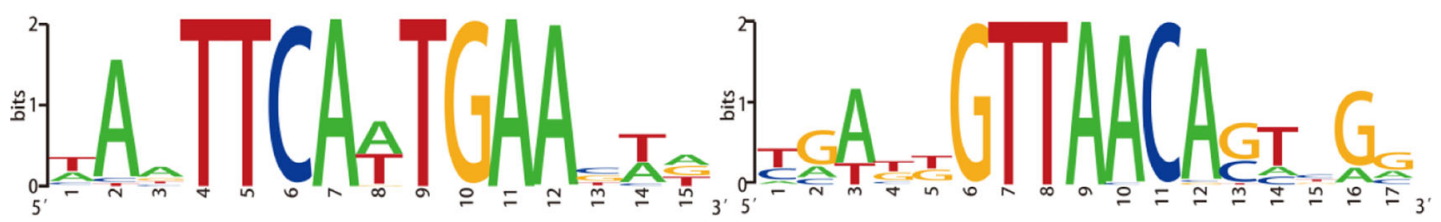

b

GO enrichment analysis

photosystem II antenna complex PSII associated light-harvesting complex IIlight-harvesting complex-

thylakoid light-harvesting complexchloroplast thylakoid membrane protein complexregulation of salicylic acid biosynthetic process Golgi apparatus nonphotochemical quenchingE regulation of salicylic acid metabolic process-

○ regulation of systemic acquired resistanceresponse to oxygen-containing compoundresponse to chemical regulation of hormone biosynthetic processpigment binding regulation of stomatal movement response to blue light photosynthesis, light harvesting in photosystem Igerm-line stem-cell niche homeostasis iron incorporation into metallo-sulfur cluster -

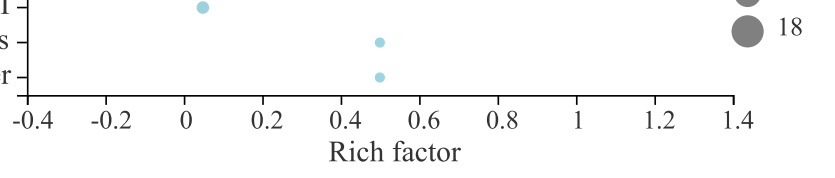

C

KEGG enrichment analysis

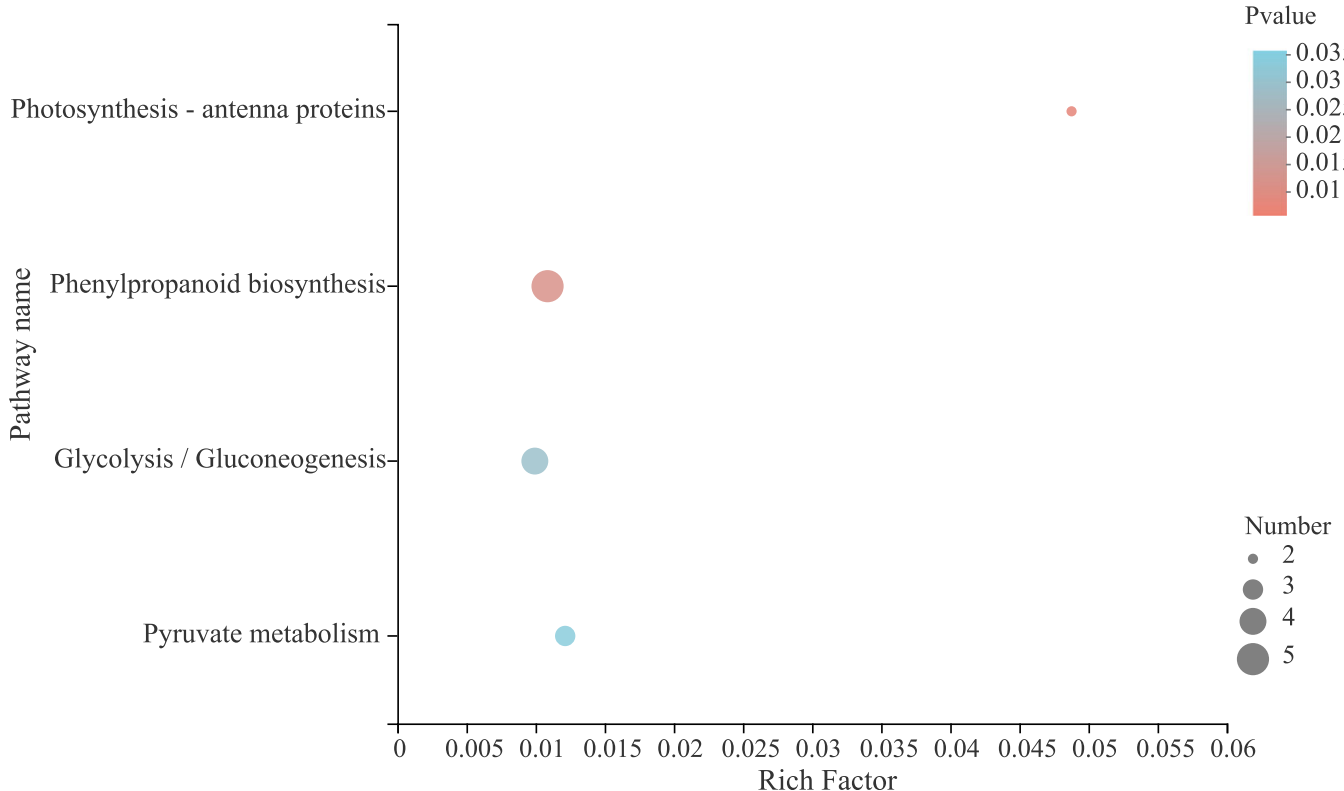

Fig. 8 (See legend on next page.) 
(See figure on previous page.)

Fig. 8 BBX binding pattern, GO and KEGG annotation. a BBX target gene prediction. The two predicted BBX binding patterns resulted in the targeting of two different sets of genes, termed target genes. $\mathbf{b}$ GO enrichment analysis of BBX target genes from the enrichment top 20. The vertical axis represents the GO term, and the horizontal axis represents the Rich factor, with larger Rich factor indicating greater enrichment. The size of the dots indicates the number of genes in the $\mathrm{GO}$ term, and the color of the dots corresponds to different $P$-value ranges. The top 20 enrichment results are displayed for $P$-value $<0.05$. c)KEGG enrichment analysis of BBX target genes from the top 20. The vertical axis represents the pathway name, the horizontal axis represents the Rich factor and the size of the points indicates the number of genes in the pathway. The top 20 enrichment results are displayed for $P$-value $<0.05$

(OsBBX14-OX) delayed heading date by repressing the expression of the flowering-forming factor gene under long-day (LD) and short-day (SD) conditions [40]. Based on studies in the subclade IV of Arabidopsis and rice, it is hypothesized that the expansion of the subclade IV of $P e B B X$ may have an effect on flowering and photomorphogenesis and development phenomena in Moso bamboo.

The motif analysis revealed that different BBX proteins in subclades I, II and IV contain motifs with unclear functions. In addition, there were three BBX proteins (PeBBX02, PeBBX19, PeBBX24) had a valine-proline (VP) motif consisting of 6 amino acids at the Cterminus, with a consistent sequence of G-I/V-V-V-P-S/ T-F (Additional file 3: Figure S3). The VP motif is generally about 16-20 amino acid residues away from the CCT structural domain, which plays a vital role in the interaction between BBX protein and coiled-coil proteins $[41,42]$. COP1 (CONSTITUTIVE PHOTOMORPHOGENIC 1) is involved in the degradation of HY5 as a pan-peptide ligase component and inhibits plant photomorphogenesis [43]. Additionally, it was previously shown that different VP motifs allow different BBXs to bind the WD40 (Trp-Asp-40) structural domain of COP1 with different affinities, thereby regulating photomorphogenesis [44].

The amino acid sequences of B-box1 and B-box2 structural domains in animals is significantly different compared to plants, but within plants these domains are much more consistent. In addition, the evolutionary trajectory of $\mathrm{BBX}$ domains in green plants indicates that the original $\mathrm{BBX}$ protein had only one $\mathrm{BBX}$ domain, which underwent a duplication event later in evolution. After this event, the CCT domain was formed. The deletion of the 2 BBX and CCT structural domains during the subsequent evolution, as well as the further replication events of the BBX structural domain, helped BBX proteins expand into different structural types [7]. PeBBX09 had only one BOX1 domain, presumably due to a deletion that occurred during evolution.

Gene duplication has been shown to be a significant factor in the evolution of genes with novel functions [45]. In the process of species evolution, the duplication events can promote the evolution of species and the rapid expansion of the species genome, and most of the duplication events occur in species due to the drastic changes in the survival environment [46]. Furthermore, during the growth and development of species, gene duplication events mainly help species to adapt to different environments, thus adapting to the drastically changing external environment and increasing the survival rate of the species [47, 48]. Jiao et al. [49] showed that one of the gene duplication events in plants occurred 192 mya. In addition, it has been reported that the Moso bamboo SPL gene family had a large duplication event at about 15 mya [26]. Among the gene pairs identified, PeBBX14/ PeBBX16, PeBBX17/PeBBX05, PeBBX18/PeBBX03, PeBBX19/PeBBX02, PeBBX21/PeBBX12, PeBBX23/ PeBBX27, PeBBX06/PeBBX09 and PeBBX08/PeBBX11 occurred between 9 and 15 mya, close to the previously reported large-scale duplication events of 7-15 mya for the whole genome of Mao bamboo [26].

In gene family evolution and expansion, tandem duplication and segmental duplication occur frequently [46]. Zhang et al.[50] found that fragment duplication was the main driving force for the expansion of the RPD3 gene family in cotton in a covariance analysis. In the Moso bamboo $B B X$ gene family, no tandem gene clusters were

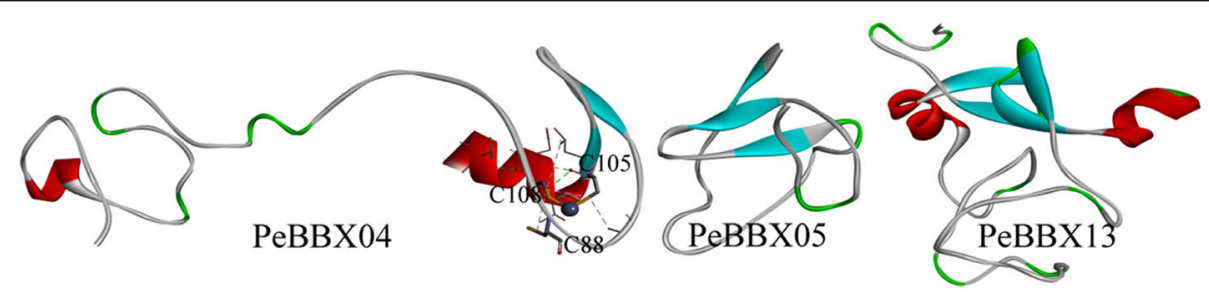

Fig. 9 Three-dimensional structure of the Moso bamboo BBX protein sequences. Models were constructed with the SWISS-MODEL software. The red areas indicate the $a$-helices and the bright blue areas represent the $\beta$-folds. C88, C105 and C108 indicate cysteines bound to a zinc ion 
a
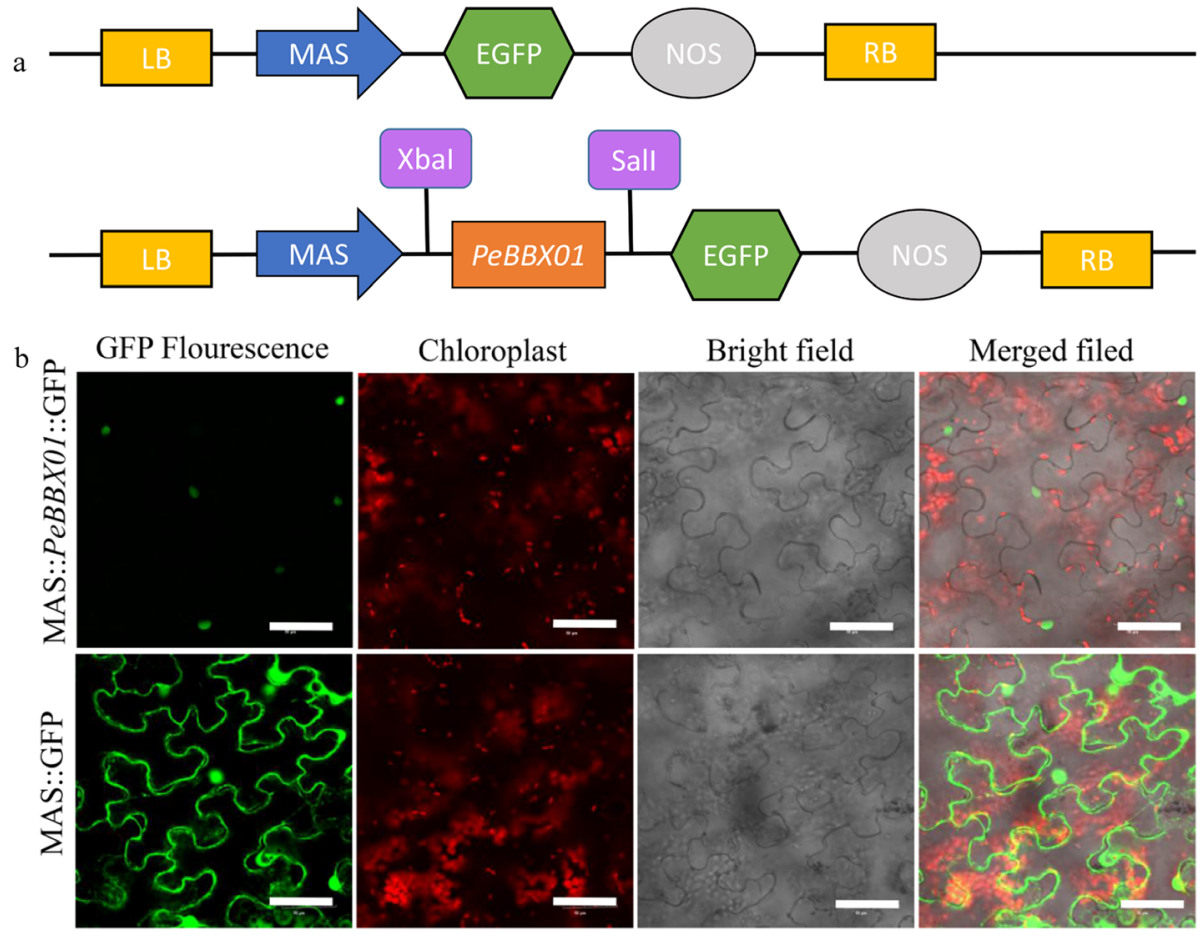

Merged filed

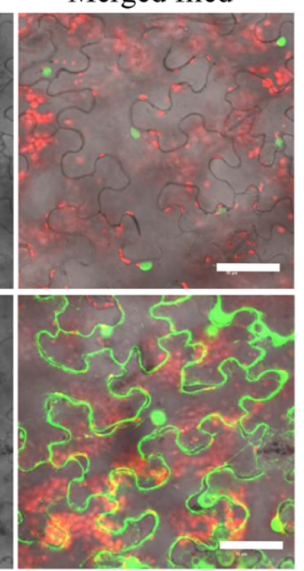

Fig. 10 Subcellular localization of the GFP-fused PeBBX protein. a Schematic drawing of PeBBX01 subcellular localization. b Subcellular localization of PeBBX01. The fusion protein MAS-PeBBX01-GFP and the control vector were transiently expressed in tobacco leaves and then observed by fluorescence microscopy. Scale bar was $50 \mu \mathrm{m}$

identified, and 24 gene pairs were identified, suggesting that segmental duplication events may be more important than tandem duplication in the expansion of the $P e B B X$ genes. Interestingly, two genes of these homologous gene pairs belonged to the same subclade, suggesting that some BBX family genes might arise from gene duplication events and that these duplication events were the main drivers of gene family expansion [51]. As a result of interspecies collinearity analysis, some homologous regions were formed between different chromosomes. Some PeBBX genes fell within these homologous regions, suggesting that genome-wide amplification of the $B B X$ gene family also occurred. The expansion of BBX during evolution and their high conservation throughout different plant species suggest that BBX may play an essential role in the adaptation of plants to terrestrial environments $[52,14]$.

Plant hormones were originally described as regulators during growth and development, acting as signaling molecules to regulate complex metabolic pathways [53, 54]. In a previously study, four tomato BBX genes were induced by ethylene $(\mathrm{ETH})$ and all of them had ERE in their promoters [2]. In grapes, four BBX genes expression $(V v B B X 2, V v B B X 3, V v B B X 13$ and $V v B B X 22)$ were affected by $\mathrm{ABA}$ and $\mathrm{ETH}$, and the promoter regions of all four genes contained ABRE promoter elements [55]. Three $P h B B X$ genes (PhBBX8, PhBBX15 and $P h B B X 20)$ contained drought-responsive cis-elements were affected by drought in Petunia hybrida [56]. Moso bamboo has the property of explosive growth, which can grow about $20 \mathrm{~m}$ in 1.5 months [57]. It was found that the growth of Moso bamboo was correlated with GA. Exogenous gibberellin (GA3) was applied to Moso bamboo seedlings and it was observed to significantly increase internode length [58]. In the currently study, there were 9 genes containing ERE promoter elements and 14 genes contained drought response elements (MBS), and almost all of them contained ABRE. In addition, a large number of light response elements, hormone response elements and abiotic stress response elements were distributed in the promoters of $P e B B X \mathrm{~s}$, suggesting that these genes might play essential functions in light signaling, hormone and stress response.

There were studies have shown that growth regulation effect of BBX [59]. AtBBX24 binded to DELLA protein and prevented DELLA-mediated inhibition of PIF4 activity, which in turn PIF4 binded to the promoter of cell elongation-related genes to promote cell elongation [60]. It has been shown that expression of apple $M d B B X 10$ in Escherichia coli enhanced cellular tolerance to salt and osmotic stress, and overexpression of this gene in Arabidopsis also enhanced the tolerance of transgenic plants to abiotic stresses, such as drought and salt [61, 62]. High salt and polyethylene glycol (PEG) treatment- 
induced $S s B B X 24$ gene expression and protein accumulation in potato (Solanum sogarandinum), and the length of daylight also regulated the response of $S s B B X 24$ to salt stress [63]. In addition to direct regulation at the transcriptional and protein levels, BBX proteins have been shown to play important roles in hormone signaling by growth hormones, such as indole-3-acetic acid (IAA), gibberellic acid (GA), ABA and brassinolide (brassinosteroid, BR) [64]. $A t B B X 21$ acts as a negative regulator related to growth hormones, ETH and BR, thus affecting plant growth under long-term shade conditions [65]. Additionally, GA may be involved in the regulation of flowering in Dendranthema morifolium by $C m B B X 24$ [22]. Additionally, our expression analysis indicated that $P e B B X$ genes might play an important role in the process of plant growth and development, different $P e B B X$ genes had expression specificity at different heights.

Protein interaction networks contribute to the understanding of complex biological network systems [66]. Recent studies have revealed that the plant BBX structural domain plays an important role in mediating protein interactions and regulation of gene expression. In plants, the $\mathrm{BBX}$ domain functions by forming heterodimers within the BBX protein family. In Arabidopsis, AtBBX24 and $A t B B X 25$ have been shown to interfere with the function of HY5 (ELONGATED HYPOCOTYL 5) by forming an inactive heterodimeric form, which in turn affected AtBBX22/LZF1 (LIGHT-REGULATED ZINC FINGER PROTEIN 1) expression and inhibited the photomorphogenesis of seedlings [10]. Arabidopsis BBOX32 interacts with BBX4/CONSTANS-LIKE3 (COL3) to regulate flowering [67].

In addition, BBX proteins from different plants can also have cross-species effects. For example, the Nterminal BBX region of AtBBX32 in Arabidopsis has been shown to interact with the BBX protein of GmBBX62 in soybean (Glycine max) [12]. Protein interaction network analysis speculated that PeBBX proteins interact and PeBBX01 interacted with multiple proteins and it might play important roles within the Moso bamboo family.

$\mathrm{BBX}$, as a transcription factor, can regulate other proteins and thus affect plant growth and development. For example, $C m B B X 8$ accelerated flowering in chrysanthemums by directly targeting CmFLT1, a flower-inducible gene [68]. BBX16 promoted the growth of shade hypocoty possibly as a positive transcriptional regulator of PIL1 [65]. Enrichment analysis of the top 20 BBX target genes predicted that many were involved in the photosynthetic pathway, such as the PSII antenna complex, PSII associated light-harvesting complex II, lightharvesting complex and others. It has been found that PSII is the first complex involved in oxygenic photosynthesis [69]. PSII is a multisubunit pigment-protein complex that catalyzes light-driven water oxidation and reduction of plastid quinones. The light-trapping chlorophyll a/b binding protein is the light-carrying protein of the PS II light-trapping complex. Photons captured by the light-trapping chlorophyll a/b-binding antenna complex light-catching complex II (LHCII) are converted by photosynthesis into biochemical energy and biomass for regulating plant growth, development and morphogenesis [70-73].

The phenylpropanoid/flavonoid biosynthesis pathway is an important metabolic pathway in plants. For example, plant defense responses are associated with activation of the general phenylpropanoid pathway and anthocyanins are the end product of a branch of the phenylpropanoid/flavonoid biosynthesis pathway [74, 75]. It has been shown that BBX proteins not only play an essential role in regulating photomorphogenesis [76], but also light-induced anthocyanin synthesis. It was previously demonstrated that in Arabidopsis, HY5 is a core regulator that facilitates the photomorphogenesis process and that $A t B B X 32$ can bind to $A t B B X 21$ and then interact with $\mathrm{HY} 5$ to reduce its transcriptional activity $[77,78]$. In contrast, $A t B B X 24$ might interfere with the binding of HY5 to the promoter of anthocyanin genes by forming a heterodimer with HY5 [79]. PpBBX16 in pear (Pyrus pyrifolia) has also been shown to be a positive regulator of anthocyanin in berries [80]. The enrichment analysis of target genes of BBX were also closely related to the carbohydrate metabolism pathway. It has been described previously that carbohydrates play an important role in plant growth and stress tolerance $[81,82]$. Overall, the analysis of the KEGG metabolic pathway led to a better understanding of the target genes and the pathways regulated by $P e B B X$.

\section{Conclusions}

In this study, 27 members of the Moso bamboo BBX family were examined and divided into three subclades based on conserved domains. Some fragment duplications were found on different chromosomes, which likely drove the expansion of the $P e B B X$ gene family. Bioinformatic analysis of gene structure, conserved motifs, chromosomal position, covariance and cis-element prediction greatly expanded the current understanding of the structural relationships among family members. Target gene prediction of $P e B B X$ genes resulted in the identification of several key functional genes, opening up a number of new avenues for exploring the role of these genes in Moso bamboo development.

\section{Materials and methods}

Identification of $B B X$ genes in the $P$. edulis genome

The Moso bamboo genome was downloaded from GigaDB (http://gigadb.org/dataset/100498). In order to 
identify the BBX TF genes in P. edulis, the BBX domain Hidden Markov Model (HMM) was used as a query to search the Moso bamboo genome. After removing redundant hits and initial chromosomal localization, BBX family genes were identified by further analysis using CDD (http://www.ncbi.nlm.nih.gov/Structure/cdd/ wrpsb.cgi) to reveal potential non-redundant BBX TF genes, termed $P e B B X$ genes. The Molecular weight $(\mathrm{MW})$ and isoelectric point (pI) of the deduced amino acid sequences were predicted with the ExPASy online tool. Next, the SignalP 4.1 Server (http://www.cbs.dtu. $\mathrm{dk} /$ services/SignalP/) was used to predict signal peptides of each member of the BBX family [83, 84]. Finally, Plant-mPLoc was employed to predict the subcellular localization of proteins [85].

\section{Phylogenetic analysis}

The BBX protein sequences of Arabidopsis and rice were downloaded from the Arabidopsis Information Resource, version 10 (TAIR 10) (http://www.arabidopsis.org) and the RGAP 7 database (Rice Genome Annotation Project, http://rice.plantbiology.msu.edu/). The BBX protein sequences from $A$. thaliana, Oryza sativa and $P$. edulis were used to construct a phylogenetic tree using the neighbor-joining (NJ) method. For statistical reliability, the nodes of the tree were assessed by bootstrap analysis containing 1000 replicates.

\section{Analysis of gene structure, motifs, domains and multi- sequence alignments}

To better understand the structures of the $P e B B X$ genes, their exon-intron information was obtained from the whole genome GFF annotation file, then visualized with TBtools. To better characterize the structures of the PeBBX proteins, the software Multiple Expectation Maximization for Motif Elicitation (MEME) was used to identify conserved motifs [86]. The following optimization parameters were used: any number of repeats, optimum width of each motif restricted between 6 and 50 residues and a maximum number of 5 motifs. Alignments of protein sequences and the corresponding residues were carried out using Geneious software with embedded parameters set to cost matrix: Blosum62. In addition, conserved domain analysis was performed using Conserved Domain Search (https://www.ncbi.nlm. nih.gov/Structure/cdd/wrpsb.cgi).

\section{Cis-acting elements in the $P e B B X$ gene promoter regions}

In order to identify conserved cis-elements within the promoter regions of the $P e B B X$ genes, the sequences 1500 bp upstream of the initiation codons were considered as the proximal promoter region sequences. These sequences were submitted to the PlantCARE database for promoter prediction analysis (http://bioinformatics. psb.ugent.be/webtools/plantcare/html/), followed by functional categorization and visualization using TBtools [87].

\section{Chromosomal locations, genomic duplications and $\mathrm{Ka} / \mathrm{Ks}$ ratios}

To identify the locations of the $P e B B X$ members on the Moso bamboo chromosomes, the termination and start positions of the coding sequences (CDSs) were retrieved from the Moso bamboo database. Each $P e B B X$ gene was placed on the corresponding Moso bamboo chromosome according to the physical location of the gene and schematically mapped through the MapChart online website. Circos software was used to visualize the gene duplications of Moso bamboo PeBBX genes [88]. Sequences from rice, Arabidopsis, pepper and maize were identified by BLAST comparison to determine homologous gene pairs [89]. Then, MCScanX was used to identify homologous regions [90].

The ratio of non-synonymous substitutions rate (Ka)/ synonymous substitutions rate (Ks) was used to determine the homologous relationship and divergence time of $\mathrm{PeBBX}$ genes. For $\mathrm{Ka} / \mathrm{Ks}$ analysis, 24 homologous gene pairs were identified by BLAST. $\mathrm{Ka} / \mathrm{Ks}$ ratios between homologous gene pairs were calculated using KaKs_Calculator 2.0 [91]. The evolutionary divergence time within the $P e B B X$ genes was computed using the bamboo-specific divergence time formula $\mathrm{T}=\mathrm{Ks} / 2 \lambda(\lambda=$ $\left.6.5 \times 10^{-9}\right)[24]$.

\section{Expression patterns of $P e B B X$ genes}

To analyze the expression patterns of the $P e B B X$ genes and identify genes that may be related to rapid shoot development, we downloaded RNA-seq data (Accession: SRX3282084, SRX3282085, SRX3282086, SRX3282087, SRX3282088, SRX3282089, SRX3282090, SRX3282091, SRX3282092, SRX3282093, SRX3282094, SRX3282095, SRX3282096, SRX3282097, SRX3282098, SRX3282099, SRX3282100, SRX3282101, SRX3282102, SRX3282103, SRX3282104, SRX3282105, SRX3282106, SRX3282107) from the gene expression profile database of NCBI (http://www.ncbi.nlm.nih.gov/geo/). Transcriptomic data with replicates came from bamboo shoots at different growth stages $(0.2 \mathrm{~m}, 0.5 \mathrm{~m}, 1 \mathrm{~m}, 2 \mathrm{~m}, 3 \mathrm{~m}, 5 \mathrm{~m}, 6$ and $7 \mathrm{~m})$. Transcriptome data which was quantified as transcripts per million reads (TPM) were log2-transformed [92] and imported into TBtools, where Amazing Heatmap was used to generate expression heatmaps. For time-series gene expression analysis, we further employed the STEM algorithm with a number of timeseries patterns of 10 and a significant trend P-value set to 0.001 . 
Protein-protein interaction (PPI) network construction PPI relationships were analyzed using protein sequences from PeBBX members. Prediction of PPI networks was performed using the Retrieval of Interacting Genes/Proteins online database (STRING v10) (http://string-db. org) [93]. Cytoscape is an open-source bioinformatics software platform used for visualizing molecular interaction networks [94], which we employed to build PPI network maps. The minimum required score for the interaction was set to $\geq 0.15$ when constructing the interaction network.

\section{Plant Material, RNA extraction and quantitative real-time (qRT-PCR) analysis}

In order to analyze the tissue expression pattern of PeBBX genes, various growth stages samples of Moso bamboo tissues were collected from bamboo plants growing in the local Moso bamboo forests in Guilin, Guangxi (All samples collection with protocols approved by the owner), including various young parts of $0.2 \mathrm{~m}$, $0.5 \mathrm{~m}, 1 \mathrm{~m}, 2 \mathrm{~m}, 3 \mathrm{~m}, 5 \mathrm{~m}, 6$ and $7 \mathrm{~m}$. The collected samples were kept in three independent replicates and all samples were stored at $-80{ }^{\circ} \mathrm{C}$ until further experiments, followed by grinding to fine powder with a mortar and pestle.

A FastPure Plant Complete RNA Isolation Package was utilized to extract RNA from Moso bamboo samples for qRT-PCR analysis, following the manufacturer's instructions (Vazyme company, China). First-strand cDNA was synthesized with a HiScript@ lll 1st Strand cDNA Synthesis Kit (+ gDNA wiper), following the manufacturer's instruction. Gene-specific qRT-PCR primers were designed by Primer Premier 5 (See Additional file 7: Table S4) based on the CDS of each gene. Each qRTPCR experiment was conducted in triplicate using separate RNA samples. The reaction conditions were: $95{ }^{\circ} \mathrm{C}$ for $5 \mathrm{~min}$, followed by 38 cycles of $95{ }^{\circ} \mathrm{C}$ for $15 \mathrm{~s}$ and $55{ }^{\circ} \mathrm{C}$ for 15 s. A melting curve from 65 to $95{ }^{\circ} \mathrm{C}$ was then used. The actin gene was used as an internal control for normalization. Data were subjected to one-way analysis of variance (ANOVA), followed by Tukey's test using SPSS software [95].

\section{PeBBX target gene identification, annotation and expression analysis}

Gene promoter sequences (2000 bp upstream of the start site) were extracted from the genome of Moso bamboo. The JASPAR database (http://jaspar.genereg. net/) was used to predict the binding site genes of PeBBX [96]. The screening index was set to less than 1.0 $\mathrm{E}^{-6}$ to identify the genes targeted by BBX members.

Geno ontology (GO) annotation was performed using the UniProt-GOA database (www.http://www.ebi.ac.uk/ GOA/). Protein IDs were converted to UniProt IDs for
GO mapping analysis and KEGG protein pathway annotation based on the KEGG database (http://www.kegg.jp/ kegg/ko.html) [97].

\section{Protein structure analysis by homology modeling}

The three-dimensional (3D) structures of PeBBX proteins were predicted in order to analyze potential protein functions. The tertiary structures of PeBBX proteins were generated by homology modeling techniques using the online template-based SWISS-MODEL (http:// swissmodel.expasy.org) [98].

\section{Subcellular localization of BBX proteins}

To verify the localization of $P e B B X 01$ in plant cells, the $P e B B X 01$ gene was selected to design primers with a homologous recombination arm (the primers see Additional file 12), and the full-length CDS sequence with no terminator codon amplified from the cDNAs of Moso bamboo, then the fragment was cloned into the p1300GFP vector. Within this vector, the $P e B B X 01$ gene was fused with the green fluorescent protein (GFP) gene under the control of the promoter to form a recombinant vector. The accuracy of the positive clone was ensured by PCR and DNA sequencing, and it was transformed into the Agrobacterium tumefaciens strain GV3101 [99, 100],. Tobaccos (Nicotiana benthamiana) were cultivated in a greenhouse at $23{ }^{\circ} \mathrm{C}$ for about one month, then the tobacco in good growth condition was selected, and Agrobacterium tumefaciens carrying the recombinant vector was injected into the third or fourth leaf and incubated at $25^{\circ} \mathrm{C}$ for $24 \mathrm{~h}$ in the dark, followed by $24-72 \mathrm{~h}$ in the light, to make sections for observation under a laser confocal microscope (Olympus, Tokyo, Japan) [55]

\section{Abbreviations \\ TFs: Transcription factors; BBX: B-box; CCT: CONSTANS, CCMike and TOC; BBX 1: B1; BBX 2: B2; CO: CONSTAVS; FT: FLOWERING LOCUS T; MeJA: Methyl jasmonate; $A B A$ : Abscisic acid; ARE: Anaerobic response regulatory elements; LTR: Low-temperature response elements; BP: Biological process; CC: Cellular component; MF: Molecular function; PSII: Photosystem II; VP: Valine-proline; COP1: CONSTITUTIVE PHOTOMORPHOGENIC 1; PEG: Polyethylene glycol; IAA: Indole-3-acetic acid; GA: Gibberellic acid; BR: Brassinolide (brassinosteroid); HY5: ELONGATED HYPOCOTYL 5; LZF1: LIGHT-REGULATED ZINC FINGER PROTEIN 1; LHCIl: Light-catching complex II; MEME: Multiple Expectation Maximization for Motif Elicitation; CDS: Coding sequences; TPM: Transcripts per million reads; PPI: Protein-protein interaction; qRT- PCR: Quantitative real-time; STEM: Short time-series expression miner; LD: Long-day; SD: Short-day; ETH: Ethylene; GFP: Green fluorescent protein}

\section{Supplementary Information}

The online version contains supplementary material available at https://doi. org/10.1186/s12864-021-07821-w.

Additional file 1: Figure S1. The distribution of the PeBBXgenes on the scaffolds of Moso bamboo. PeBBX genes are numbered 1-27. The chromosome number is shown at the top of each strip, with the gene name displayed on the right or left side of the chromosome. 
Additional file 2: Figure S2. Location of cis-elements in the promoters of PeBBX genes. Cis-element analysis of PeBBXs. The 1500 bp DNA fragments upstream of the ATG were analyzed using the online analysis software PlantCARE. Different cis-acting elements of PeBBXgenes are displayed. The different colored markers indicate different predictedcisacting elements.

Additional file 3: Figure S3. VP motif. Valine-proline (VP) motif, the proteins with red underlines possess typical VP residues.

Additional file 4: Table S1. The IDs and sequences of BBX proteins from rice, Arabidopsisand Moso bamboo used in phylogenetic tree construction.

Additional file 5: Table S2. Cis-element analysis of PeBBX genes. Additional file 6: Table S3. The FPKM value of PeBBX genes in different heights.

Additional file 7: Table S4. The primers used for QRT-PCR.

Additional file 8. BBX target genes

Additional file 9: The founctional $\mathrm{GO}$ analysis.

Additional file 10: The founctional annotation of BBX target genes.

Additional file 11: The founctional KEGG analysis.

Additional file 12: The primers of PeBBX01.

\section{Acknowledgements}

The authors would like to thank TopEdit (www.topeditsci.com) for linguistic assistance during preparation of this manuscript. Not applicable.

\section{Guidelines or legislation statement}

The experimental study of plants, the plant material used and the collection of plant material for this experiment complied with relevant institutional, national and international guidelines and legislation. And the study complied with local and national regulations.

\section{Authors' contributions}

RFM was involved in the study design and interpretation, conducted the experiments and wrote the manuscript. JLC helped analyze the data. $\mathrm{BH}$ and $\mathrm{ZNH}$ helped revise the manuscript. ZJZ was involved in the design and interpretation of the study and refined this manuscript. ZJZ was responsible for the completeness of the data and the accuracy of the data analysis. All members were involved in the manuscript modification and all authors have read and agreed to the published version of the manuscript. The author(s) read and approved the final manuscript.

\section{Funding}

The work was funded by the National Natural Science Foundation of China (NNSFC, Project grant: 31770721). The funder had no role in study design, data collection and analysis, data interpretation or preparation of the manuscript.

\section{Availability of data and materials}

All data supporting the conclusions of this article are provided within the article and its additional files. The genomics sequences data of Moso bamboo and rice are available in the GigaDB Database (http://gigadb.org/ dataset/100498), the Arabidopsis Information Resource, version 10 (TAIR 10) (http://www.arabidopsis.org) and the RGAP 7 database (Rice Genome Annotation Project, http://rice.plantbiology.msu.edu/). The gene expression profile database of NCBI (http://www.ncbi.nlm.nih.gov/geo/), accession: SRX3282084, SRX3282085, SRX3282086, SRX3282087, SRX3282088, SRX3282089, SRX3282090, SRX3282091, SRX3282092, SRX3282093, SRX3282094, SRX3282095, SRX3282096, SRX3282097, SRX3282098, SRX3282099, SRX3282100, SRX3282101, SRX3282102, SRX3282103, SRX3282104, SRX3282105, SRX3282106, SRX3282107.

\section{Declarations}

Ethics approval and consent to participate

Not applicable. The plant material used in this study was bamboo plants growing in Guilin, Guangxi, and tissue samples of bamboo shoots of different heights were obtained. The samples was collected from private land, and all samples collection with protocols approved by the owner. Since this study did not involve any endangered or protected species, no ethical approval or consent was required.

\section{Consent for publication}

Not applicable.

\section{Competing interests}

The authors declare no conflict of interest.

Received: 5 March 2021 Accepted: 17 June 2021

Published online: 13 July 2021

\section{References}

1. Gong W, Shen YP, Ma LG, Pan Y, Du YL, Wang DH, Yang JY, Hu LD, Liu XF, Dong CX, et al. Genome-wide ORFeome cloning and analysis of Arabidopsis transcription factor genes. Plant Physiol. 2004;135(2):773-82.

2. Chu Z, Wang X, Li Y, Yu H, Li J, Lu Y, Li H, Ouyang B. Genomic Organization, Phylogenetic and Expression Analysis of the B-BOX Gene Family in Tomato. Front Plant Sci. 2016;7:1552.

3. Smita S, Katiyar A, Chinnusamy V, Pandey DM, Bansal KC. Transcriptional Regulatory Network Analysis of MYB Transcription Factor Family Genes in Rice. Front Plant Sci. 2015;6:1157.

4. Li J, Han G, Sun C, Sui N. Research advances of MYB transcription factors in plant stress resistance and breeding. Plant Signal Behav. 2019:14(8):1613131.

5. Shalmani A, Fan S, Jia P, Li G, Muhammad I, Li Y, Sharif R, Dong F, Zuo X, Li $K$ et al. Genome Identification of B-BOX Gene Family Members in Seven Rosaceae Species and Their Expression Analysis in Response to Flower Induction in Malus domestica. Molecules 2018;23(7):1763-87.

6. Khanna R, Kronmiller B, Maszle DR, Coupland G, Holm M, Mizuno T, Wu SH. The Arabidopsis B-box zinc finger family. Plant Cell. 2009;21(11):3416-20.

7. Crocco CD, Botto JF. BBX proteins in green plants: insights into their evolution, structure, feature and functional diversification. Gene. 2013;531(1): 44-52.

8. Massiah MA, Matts JA, Short KM, Simmons BN, Singireddy S, Yi Z, Cox TC. Solution structure of the MID1 B-box2 $\mathrm{CHC}(\mathrm{D} / \mathrm{C}) \mathrm{C}(2) \mathrm{H}(2)$ zinc-binding domain: insights into an evolutionarily conserved RING fold. J Mol Biol. 2007;369(1):1-10.

9. Huang J, Zhao X, Weng $X$, Wang $L$, Xie W. The rice B-box zinc finger gene family: genomic identification, characterization, expression profiling and diurnal analysis. Plos One. 2012;7(10):e48242.

10. Gangappa SN, Crocco CD, Johansson H, Datta S, Hettiarachchi C, Holm M, Botto JF. The Arabidopsis B-BOX protein BBX25 interacts with HY5, negatively regulating BBX22 expression to suppress seedling photomorphogenesis. Plant Cell. 2013;25(4):1243-57.

11. Gangappa SN, Holm M, Botto JF. Molecular interactions of BBX24 and BBX25 with HYH, HY5 HOMOLOG, to modulate Arabidopsis seedling development. Plant Signal Behav. 8(8):e25208-4.

12. Qi Q, Gibson A, Fu X, Zheng M, Kuehn R, Wang Y, Wang Y, Navarro S, Morrell JA, Jiang $D$, et al. Involvement of the N-terminal B-box domain of Arabidopsis BBX32 protein in interaction with soybean BBX62 protein. J Biol Chem. 2012;287(37):31482-93.

13. Lin F, Jiang Y, Li J, Yan T, Fan L, Liang J, Chen ZJ, Xu D, Deng XW. B-BOX DOMAIN PROTEIN28 Negatively Regulates Photomorphogenesis by Repressing the Activity of Transcription Factor HY5 and Undergoes COP1Mediated Degradation. Plant Cell. 2018;30(9):2006-19.

14. Gangappa SN, Botto JF. The BBX family of plant transcription factors. Trends Plant Sci. 2014;19(7):460-70.

15. Tiwari SB, Shen Y, Chang HC, Hou Y, Harris A, Ma SF, McPartland M, Hymus GJ, Adam L, Marion C, et al. The flowering time regulator CONSTANS is recruited to the FLOWERING LOCUS T promoter via a unique cis-element. New Phytologist. 2010;187(1):57-66.

16. Jang S, Marchal V, Panigrahi KC, Wenkel S, Soppe W, Deng XW, Valverde F, Coupland G. Arabidopsis COP1 shapes the temporal pattern of CO accumulation conferring a photoperiodic flowering response. EMBO J. 2008; 27(8):1277-88.

17. Robson F, Costa MMR, Hepworth SR, Vizir I, Pin eiro M, Reeves PH, Putterill J, Coupland G. Functional importance of conserved domains in the floweringtime gene CONSTANS demonstrated by analysis of mutant alleles and transgenic plants. Plant J. 2001;28(6):619-31. 
18. Datta S, Hettiarachchi GH, Deng XW, Holm M. Arabidopsis CONSTANS-LIKE3 is a positive regulator of red light signaling and root growth. Plant Cell. 2006;18(1):70-84.

19. Yan H, Marquardt K, Indorf M, Jutt D, Kircher S, Neuhaus G, RodriguezFranco M. Nuclear localization and interaction with COP1 are required for STO/BBX24 function during photomorphogenesis. Plant Physiol. 2011;156(4): $1772-82$.

20. Urano K, Kurihara Y, Seki M, Shinozaki K. 'Omics' analyses of regulatory networks in plant abiotic stress responses. Curr Opin Plant Biol. 2010;13(2): 132-8.

21. Hannah MA, Heyer AG, Hincha DK. A global survey of gene regulation during cold acclimation in Arabidopsis thaliana. PLoS Genet. 2005;1(2):e26.

22. Yang Y, Ma C, Xu Y, Wei Q, Imtiaz M, Lan H, Gao S, Cheng L, Wang M, Fei Z, et al. A Zinc Finger Protein Regulates Flowering Time and Abiotic Stress Tolerance in Chrysanthemum by Modulating Gibberellin Biosynthesis. Plant Cell. 2014;26(5):2038-54.

23. Chen J, Chen J-y, Wang J-n, Kuang J-f, Shan W, Lu W-j. Molecular characterization and expression profiles of MaCOL1, a CONSTANS-like gene in banana fruit. Gene. 2012;496(2):110-7.

24. Peng Z, Lu Y, Li L, Zhao Q, Feng Q, Gao Z, Lu H, Hu T, Yao N, Liu K, et al. The draft genome of the fast-growing non-timber forest species moso bamboo (Phyllostachys heterocycla). Nat Genet. 2013;45(4):456-61 461e451452.

25. Peng Z, Zhang C, Zhang Y, Hu T, Mu S, Li X, Gao J. Transcriptome sequencing and analysis of the fast growing shoots of moso bamboo (Phyllostachys edulis). PloS one. 2013;8(11):e78944.

26. Pan F, Wang Y, Liu H, Wu M, Chu W, Chen D, Xiang Y. Genome-wide identification and expression analysis of SBP-like transcription factor genes in Moso Bamboo (Phyllostachys edulis). BMC Genomics. 2017;18(1):486.

27. Tao GY, Ramakrishnan M, Vinod KK, Yrjala K, Satheesh V, Cho J, Fu Y, Zhou M. Multi-omics analysis of cellular pathways involved in different rapid growth stages of moso bamboo. Tree Physiol. 2020;40(11):1487-508.

28. Ramakrishnan M, Yrjälä K, Vinod KK, Sharma A, Cho J, Satheesh V, Zhou M. Genetics and genomics of moso bamboo (Phyllostachys edulis): Current status, future challenges, and biotechnological opportunities toward a sustainable bamboo industry. Food Energy Sec. 2020;9(4):e229-264.

29. Liu J, Cheng Z, Xie L, Li X, Gao J. Multifaceted Role of PheDof12-1 in the Regulation of Flowering Time and Abiotic Stress Responses in Moso Bamboo (Phyllostachys edulis). Int J Mol Sci. 2019;20(2):424-36.

30. Wu HL, Lv H, Li L, Liu J, Mu SH, Li XP, Gao J. Genome-Wide Analysis of the AP2/ERF Transcription Factors Family and the Expression Patterns of DREB Genes in Moso Bamboo (Phyllostachys edulis). Plos One. 2015;10(5): e0126657-22

31. Zhao H, Gao Z, Wang L, Wang J, Wang S, Fei B, Chen C, Shi C, Liu X, Zhang $\mathrm{H}$ et al. Chromosome-level reference genome and alternative splicing atlas of moso bamboo (Phyllostachys edulis). GigaScience. 2018;7(10):giy115-12.

32. Zhao H, Wang S, Wang J, Chen C, Hao S, Chen L, Fei B, Han K, Li R, Shi C et al. The chromosome-level genome assemblies of two rattans (Calamus simplicifolius and Daemonorops jenkinsiana). GigaScience. 2018;7(9):giy097-11.

33. Singh KB, Foley RC, Oñate-Sánchez L. Transcription factors in plant defense and stress responses. Curr Opin Plant Biol. 2002;5(5):430-6.

34. Yamaguchi-Shinozaki K, Shinozaki K. Organization of cis-acting regulatory elements in osmotic- and cold-stress-responsive promoters. Trends Plant Sci. 2005;10(2):88-94.

35. Talar U, Kielbowicz-Matuk A, Czarnecka J, Rorat T. Genome-wide survey of Bbox proteins in potato (Solanum tuberosum)-Identification, characterization and expression patterns during diurnal cycle, etiolation and de-etiolation. Plos One. 2017;12(5):e0177471.

36. Zou ZY, Wang RH, Wang R, Yang SL, Yang YJ. Genome-wide identification, phylogenetic analysis, and expression profiling of the BBX family genes in pear. J Hortic Sci Biotech. 2018;93(1):37-50.

37. Garg R, Patel RK, Jhanwar S, Priya P, Bhattacharjee A, Yadav G, Bhatia S, Chattopadhyay D, Tyagi AK, Jain M. Gene discovery and tissue-specific transcriptome analysis in chickpea with massively parallel pyrosequencing and web resource development. Plant Physiol. 2011;156(4):1661-78.

38. Wang S, Sun H, Xu X, Yang K, Zhao H, Li Y, Li X, Gao Z. Genome-wide identification and expression analysis of brassinosteroid action-related genes during the shoot growth of moso bamboo. Mol Biol Rep. 2019:46(2):1909-30.

39. Shou Y, Zhu Y, Ding Y. Transcriptome analysis of lateral buds from Phyllostachys edulis rhizome during germination and early shoot stages. BMC Plant Biol. 2020;20(1):229.
40. Bai B, Zhao J, Li Y, Zhang F, Zhou J, Chen F, Xie X. OsBBX14 delays heading date by repressing florigen gene expression under long and short-day conditions in rice. Plant Sci. 2016;247:25-34.

41. Holm M, Hardtke CS, Gaudet R, Deng XW. Identification of a structural motif that confers specific interaction with the WD40 repeat domain of Arabidopsis COP1. MBO J. 2001;20(1-2):118-27.

42. Datta S, Hettiarachchi C, Johansson H, Holm M. SALT TOLERANCE HOMOLOG2, a B-box protein in Arabidopsis that activates transcription and positively regulates light-mediated development. Plant Cell. 2007;19(10): 3242-55.

43. Wang H, Ma LG, Li JM, Zhao HY, Deng XW. Direct interaction of Arabidopsis cryptochromes with COP1 in light control development. Science (New York, NY). 2001;294(5540):154-8.

44. Lau K, Podolec R, Chappuis R, Ulm R, Hothorn M. Plant photoreceptors and their signaling components compete for COP1 binding via VP peptide motifs. EMBO J. 2019;38(18):e102140.

45. Xu Y, Zhang T, Li Y, Miao Z. Integrated Analysis of Large-Scale Omics Data Revealed Relationship Between Tissue Specificity and Evolutionary Dynamics of Small RNAs in Maize (Zea mays). Front Genet. 2020;11:51.

46. Cannon SB, Mitra A, Baumgarten A, Young ND, May G. The roles of segmental and tandem gene duplication in the evolution of large gene families in Arabidopsis thaliana. BMC Plant Biol. 2004;4:10

47. Boles LC, Lohmann KJ. True navigation and magnetic maps in spiny lobsters. Nature. 2003;421(6918):60-3.

48. Ferguson NM, Galvani AP, Bush RM. Ecological and immunological determinants of influenza evolution. Nature. 2003;422(6930):428-33.

49. Jiao Y, Wickett NJ, Ayyampalayam S, Chanderbali AS, Landherr L, Ralph PE, Tomsho LP, Hu Y, Liang H, Soltis PS, et al. Ancestral polyploidy in seed plants and angiosperms. Nature. 2011:473(7345):97-100.

50. Zhang J, Wu A, Wei H, Hao P, Zhang Q, Tian M, Yang X, Cheng S, Fu X, Ma $L$, et al. Genome-wide identification and expression patterns analysis of the RPD3/HDA1 gene family in cotton. BMC Genomics. 2020;21(1):643.

51. Maher C, Stein L, Ware D. Evolution of Arabidopsis microRNA families through duplication events. Genome Res. 2006;16(4):510-9.

52. Kenrick P, Crane PR. The origin and early evolution of plants on land. Nature. 1997;389(6646):33-9.

53. Pieterse CM, Van der Does D, Zamioudis C, Leon-Reyes A, Van Wees SC. Hormonal modulation of plant immunity. Annu Rev Cell Dev Biol. 2012;28: 489-521.

54. Bari $\mathrm{R}$, Jones JD. Role of plant hormones in plant defence responses. Plant Mol Biol. 2009;69(4):473-88.

55. Wei H, Wang P, Chen J, Li C, Wang Y, Yuan Y, Fang J, Leng X. Genome-wide identification and analysis of B-BOX gene family in grapevine reveal its potential functions in berry development. BMC Plant Biol. 2020;20(1):72.

56. Wen S, Zhang Y, Deng Y, Chen G, Yu Y, Wei Q. Genomic identification and expression analysis of the BBX transcription factor gene family in Petunia hybrida. Mol Biol Rep. 2020;47(8):6027-41.

57. Song X, Peng C, Zhou G, Gu H, Li Q, Zhang C. Dynamic allocation and transfer of non-structural carbohydrates, a possible mechanism for the explosive growth of Moso bamboo (Phyllostachys heterocycla). Sci Rep. 2016;6:25908.

58. Zhang $H$, Wang $H$, Zhu Q, Gao $Y$, Wang $H$, Zhao L, Wang $Y, X i F$, Wang $W$, Yang $Y$, et al. Transcriptome characterization of moso bamboo (Phyllostachys edulis) seedlings in response to exogenous gibberellin applications. BMC Plant Biol. 2018;18(1):125.

59. Shalmani A, Jing XQ, Shi Y, Muhammad I, Zhou MR, Wei XY, Chen QQ, Li WQ, Liu WT, Chen KM. Characterization of B-BOX gene family and their expression profiles under hormonal, abiotic and metal stresses in Poaceae plants. BMC Genomics. 2019;20(1):27.

60. Crocco CD, Locascio A, Escudero CM, Alabadi D, Blazquez MA, Botto JF. The transcriptional regulator BBX24 impairs DELLA activity to promote shade avoidance in Arabidopsis thaliana. Nat Commun. 2015;6:6202

61. Liu X, Dai Y, Li R, Yuan L, Chen X, Wang X. Members of B-box Protein Family from Malus domestica Enhanced Abiotic Stresses Tolerance in Escherichia coli. Mol Biotechnol. 2019;61(6):421-6.

62. Liu X, Li R, Dai Y, Yuan L, Sun Q, Zhang S, Wang X. A B-box zinc finger protein, MdBBX10, enhanced salt and drought stresses tolerance in Arabidopsis. Plant Mol Biol. 2019;99(4-5):437-47.

63. Kielbowicz-Matuk A, Rey P, Rorat T. Interplay between circadian rhythm, time of the day and osmotic stress constraints in the regulation of the expression of a Solanum Double B-box gene. Ann Bot. 2014;113(5):831-42. 
64. Vaishak KP, Yadukrishnan P, Bakshi S, Kushwaha AK, Ramachandran H, Job N, Babu D, Datta S. The B-box bridge between light and hormones in plants. J Photochem Photobiol B Biol. 2019;191:164-74.

65. Crocco CD, Holm M, Yanovsky MJ, Botto JF. Function of B-BOX under shade. Plant Signal Behav. 2011;6(1):101-4.

66. Anukriti, Dhasmana A, Uniyal S, Somvanshi P, Bhardwaj U, Gupta M, Haque S, Lohani M, Kumar D, Ruokolainen J et al: Investigation of Precise Molecular Mechanistic Action of Tobacco-Associated Carcinogen 'NNK Induced Carcinogenesis: a System Biology Approach. Genes (Basel). 2019;10(8):564-85

67. Tripathi P, Carvallo M, Hamilton EE, Preuss S, Kay SA. Arabidopsis B-BOX32 interacts with CONSTANS-LIKE3 to regulate flowering. Proc Natl Acad Sci. 2017;114(1):172-7.

68. Wang L, Sun J, Ren L, Zhou M, Han X, Ding L, Zhang F, Guan Z, Fang W, Chen $\mathrm{S}$, et al. CmBBX8 accelerates flowering by targeting CmFTL1 directly in summer chrysanthemum. Plant Biotechnol J. 2020;18(7):1562-72.

69. Crepin A, Santabarbara S, Caffarri S. Biochemical and Spectroscopic Characterization of Highly Stable Photosystem II Supercomplexes from Arabidopsis. J Biol Chem. 2016;291(36):19157-71.

70. Peng L, Ma J, Chi W, Guo J, Zhu S, Lu Q, Lu C, Zhang L. LOW PSII ACCUMULATION1 is involved in efficient assembly of photosystem II in Arabidopsis thaliana. Plant Cell. 2006;18(4):955-69.

71. Liu R, Xu YH, Jiang SC, Lu K, Lu YF, Feng XJ, Wu Z, Liang S, Yu YT, Wang XF, et al. Light-harvesting chlorophyll a/b-binding proteins, positively involved in abscisic acid signalling, require a transcription repressor, WRKY40, to balance their function. J Exp Bot. 2013;64(18):5443-56.

72. Pietrzykowska M, Suorsa M, Semchonok DA, Tikkanen M, Boekema EJ, Aro EM, Jansson S. The light-harvesting chlorophyll a/b binding proteins Lhcb1 and Lhcb2 play complementary roles during state transitions in Arabidopsis. Plant Cell. 2014;26(9):3646-60

73. Fu J, Luo Y, Sun P, Gao J, Zhao D, Yang P, Hu T. Effects of shade stress on turfgrasses morphophysiology and rhizosphere soil bacterial communities. BMC Plant Biol. 2020;20(1):92

74. Qi Y, Gu C, Wang X, Gao S, Li C, Zhao C, Li C, Ma C, Zhang Q. Identification of the Eutrema salsugineum EsMYB90 gene important for anthocyanin biosynthesis. BMC Plant Biol. 2020;20(1):186.

75. Yang LT, Zhou YF, Wang YY, Wu YM, Ye X, Guo JX, Chen LS. Magnesium Deficiency Induced Global Transcriptome Change in Citrus sinensis Leaves Revealed by RNA-SEq. Int J Mol Sci. 2019;20(13):3129-49.

76. Yadav A, Bakshi S, Yadukrishnan P, Lingwan M, Dolde U, Wenkel S, Masakapalli SK, Datta S. The B-Box-Containing MicroProtein miP1a/BBX31 Regulates Photomorphogenesis and UV-B Protection. Plant Physiol. 2019; 179(4):1876-92.

77. Holtan HE, Bandong S, Marion CM, Adam L, Tiwari S, Shen Y, Maloof JN, Maszle DR, Ohto MA, Preuss S, et al. BBX32, an Arabidopsis B-Box protein, functions in light signaling by suppressing HY5-regulated gene expression and interacting with STH2/BBX21. Plant Physiol. 2011;156(4):2109-23.

78. Gangappa SN, Botto JF. The Multifaceted Roles of HY5 in Plant Growth and Development. Mol Plant. 2016;9(10):1353-65

79. Job N, Yadukrishnan P, Bursch K, Datta S, Johansson H. Two B-Box Proteins Regulate Photomorphogenesis by Oppositely Modulating HY5 through their Diverse C-Terminal Domains. Plant Physiol. 2018;176(4):2963-76.

80. Bai S, Tao R, Tang Y, Yin L, Ma Y, Ni J, Yan X, Yang Q, Wu Z, Zeng Y, et al. BBX16, a B-box protein, positively regulates light-induced anthocyanin accumulation by activating MYB10 in red pear. Plant Biotechnol J. 2019; 17(10):1985-97.

81. Chen L, Huang Y, Xu M, Cheng Z, Zhang D, Zheng J. iTRAQ-Based Quantitative Proteomics Analysis of Black Rice Grain Development Reveals Metabolic Pathways Associated with Anthocyanin Biosynthesis. Plos One. 2016;11(7):e0159238.

82. Habermann E, Dias de Oliveira EA, Contin DR, San Martin JAB, Curtarelli L, Gonzalez-Meler MA, Martinez CA. Stomatal Development and Conductance of a Tropical Forage Legume Are Regulated by Elevated [CO2] Under Moderate Warming. Front Plant Sci. 2019;10:609

83. Chou K-C, Shen H-B. Cell-PLoc 2.0: an improved package of web-servers for predicting subcellular localization of proteins in various organisms. Nat Sci. 2010;02(10):1090-103.

84. Petersen TN, Brunak S, von Heijne G, Nielsen H. SignalP 4.0: discriminating signal peptides from transmembrane regions. Nat Methods. 2011;8(10):785-6.

85. Chou KC, Shen HB. Plant-mPLoc: a top-down strategy to augment the power for predicting plant protein subcellular localization. Plos One. 2010; 5(6):e11335.
86. Bailey TL, Williams N, Misleh C, Li WW. MEME: discovering and analyzing DNA and protein sequence motifs. Nucleic Acids Res. 2006;34:W369-373 (Web Server issue)

87. Chen C, Chen H, Zhang Y, Thomas HR, Frank MH, He Y, Xia R. TBtools: An Integrative Toolkit Developed for Interactive Analyses of Big Biological Data. Mol Plant. 2020;13(8):1194-202.

88. Krzywinski M, Schein J, Birol I, Connors J, Gascoyne R, Horsman D, Jones SJ, Marra MA. Circos: an information aesthetic for comparative genomics. Genome Res. 2009;19(9):1639-45.

89. Altschul SF, Gish W, Miller W, Myers EW, Lipman DJ. Basic local alignment search tool. J Mol Biol. 1990;215(3):403-10.

90. Wang Y, Tang H, Debarry JD, Tan X, Li J, Wang X, Lee TH, Jin H, Marler B, Guo H, et al. MCScanX: a toolkit for detection and evolutionary analysis of gene synteny and collinearity. Nucleic Acids Res. 2012;40(7):e49.

91. Wang DP, Wan HL, Zhang S, Yu J. Gamma-MYN: a new algorithm for estimating $\mathrm{Ka}$ and $\mathrm{Ks}$ with consideration of variable substitution rates. Biol Direct. 2009;4:20.

92. Cushion MT, Ashbaugh A, Hendrix K, Linke MJ, Tisdale N, Sayson SG, Porollo A. Gene Expression of Pneumocystis murina after Treatment with Anidulafungin Results in Strong Signals for Sexual Reproduction, Cell Wall Integrity, and Cell Cycle Arrest, Indicating a Requirement for Ascus Formation for Proliferation. Antimicrobial Agents Chemother. 2018;62(5): e02513-17.

93. Szklarczyk D, Franceschini A, Wyder S, Forslund K, Heller D, Huerta-Cepas J, Simonovic M, Roth A, Santos A, Tsafou KP, et al. STRING v10: protein-protein interaction networks, integrated over the tree of life. Nucleic Acids Res. 2015;43(Database issue):D447-452.

94. Shannon P, Markiel A, Ozier O, Baliga NS, Wang JT, Ramage D, Amin N, Schwikowski B, Ideker T. Cytoscape: A Software Environment for Integrated Models of Biomolecular Interaction Networks. Cold Spring Harbor Lab Press. 2003;13(11):2498-504.

95. Li J, Liu X, Wang Q, Huangfu J, Schuman MC, Lou Y. A Group D MAPK Protects Plants from Autotoxicity by Suppressing Herbivore-Induced Defense Signaling. Plant Physiol. 2019;179(4):1386-401.

96. Han J, Liu Y, Zhen F, Yuan W, Zhang W, Song X, Dong F, Yao R, Qu X. STAT3 Regulates miR-384 Transcription During Th17 Polarization. Front Cell Dev Biol. 2019;7:253.

97. Kanehisa M, Sato Y, Kawashima M, Furumichi M, Tanabe M. KEGG as a reference resource for gene and protein annotation. Nucleic Acids Res. 2016;44(D1):D457-462

98. Arnold K, Bordoli L, Kopp J, Schwede T. The SWISS-MODEL workspace: a web-based environment for protein structure homology modelling. Bioinformatics. 2006;22(2):195-201.

99. Feng Y, Wei R, Liu A, Fan S, Che J, Zhang Z, Tian B, Yuan Y, Shi G, Shang H. Genome-wide identification, evolution, expression, and alternative splicing profiles of peroxiredoxin genes in cotton. Peerj. 2021;9:e10685.

100. Wang M, Xu ZC, Ding AM, Kong YZ: Genome-Wide Identification and Expression Profiling Analysis of the Xyloglucan Endotransglucosylase/ Hydrolase Gene Family in Tobacco (Nicotiana tabacum L.). Genes-Basel. 2018;9(6):273-94.

\section{Publisher's Note}

Springer Nature remains neutral with regard to jurisdictional claims in published maps and institutional affiliations.

Ready to submit your research? Choose BMC and benefit from:

- fast, convenient online submission

- thorough peer review by experienced researchers in your field

- rapid publication on acceptance

- support for research data, including large and complex data types

- gold Open Access which fosters wider collaboration and increased citations

- maximum visibility for your research: over $100 \mathrm{M}$ website views per year

At $\mathrm{BMC}$, research is always in progress.

Learn more biomedcentral.com/submissions 